\title{
CO destruction in protoplanetary disk midplanes: Inside versus outside the CO snow surface
}

\author{
Arthur D. Bosman ${ }^{1}$, Catherine Walsh ${ }^{2}$, and Ewine F. van Dishoeck ${ }^{1,3}$ \\ ${ }^{1}$ Leiden Observatory, Leiden University, PO Box 9513, 2300 RA Leiden, The Netherlands \\ e-mail: bosman@strw. leidenuniv.nl \\ ${ }^{2}$ School of Physics and Astronomy, University of Leeds, Leeds, LS2 9JT, UK \\ ${ }^{3}$ Max-Planck-Insitut für Extraterrestrische Physik, Gießenbachstrasse 1, 85748 Garching, Germany
}

Received 25 May 2018 / Accepted 5 August 2018

\begin{abstract}
Context. The total gas mass is one of the most fundamental properties of disks around young stars, because it controls their evolution and their potential to form planets. To measure disk gas masses, $\mathrm{CO}$ has long been thought to be the best tracer as it is readily detected at (sub)mm wavelengths in many disks. However, inferred gas masses from CO in recent ALMA observations of large samples of disks in the 1-5 Myr age range seem inconsistent with their inferred dust masses. The derived gas-to-dust mass ratios from CO are between one and two orders of magnitude lower than the ISM value of $\sim 100$ even if photodissociation and freeze-out are included. In contrast, Herschel measurements of hydrogen deuteride line emission of a few disks imply gas masses in line with gas-to-dust mass ratios of 100. This suggests that at least one additional mechanism is removing $\mathrm{CO}$ from the gas phase.

Aims. Here we test the suggestion that the bulk of the $\mathrm{CO}$ is chemically processed and that the carbon is sequestered into less volatile species such as $\mathrm{CO}_{2}, \mathrm{CH}_{3} \mathrm{OH}$, and $\mathrm{CH}_{4}$ in the dense, shielded midplane regions of the disk. This study therefore also addresses the carbon reservoir of the material which ultimately becomes incorporated into planetesimals.

Methods. Using our gas-grain chemical code, we performed a parameter exploration and follow the $\mathrm{CO}$ abundance evolution over a range of conditions representative of shielded disk midplanes.

Results. Consistent with previous studies, we find that no chemical processing of CO takes place on 1-3 Myr timescales for low cosmic-ray ionisation rates, $<5 \times 10^{-18} \mathrm{~s}^{-1}$. Assuming an ionisation rate of $10^{-17} \mathrm{~s}^{-1}$, more than $90 \%$ of the CO is converted into other species, but only in the cold parts of the disk below $30 \mathrm{~K}$. This order of magnitude destruction of $\mathrm{CO}$ is robust against the choice of grain-surface reaction rate parameters, such as the tunnelling efficiency and diffusion barrier height, for temperatures between 20 and $30 \mathrm{~K}$. Below $20 \mathrm{~K}$ there is a strong dependence on the assumed efficiency of $\mathrm{H}$ tunnelling.

Conclusions. The low temperatures needed for $\mathrm{CO}$ chemical processing indicate that the exact disk temperature structure is important, with warm disks around luminous Herbig stars expected to have little to no CO conversion. In contrast, for cold disks around sun-like $\mathrm{T}$ Tauri stars, a large fraction of the emitting CO layer is affected unless the disks are young $(<1 \mathrm{Myr})$. This can lead to inferred gas masses that are up to two orders of magnitude lower. Moreover, unless $\mathrm{CO}$ is locked up early in large grains, the volatile carbon composition of the icy pebbles and planetesimals forming in the midplane and drifting to the inner disk will be dominated by $\mathrm{CH}_{3} \mathrm{OH}$, $\mathrm{CO}_{2}$ and/or hydrocarbons.
\end{abstract}

Key words. astrochemistry - protoplanetary disks - molecular processes - ISM: molecules

\section{Introduction}

The total gas mass is one of the most fundamental parameters that influences protoplanetary disk evolution and planet formation. Interactions of the gas and dust set the efficiency of grain-growth and planetesimal formation (e.g. Weidenschilling 1977; Brauer et al. 2008; Birnstiel et al. 2010; Johansen et al. 2014), while interactions of planets with the gaseous disk leads to migration of the planet and gap formation (see, e.g. Kley \& Nelson 2012; Baruteau et al. 2014, for reviews). Significant amounts of gas are needed to make giant Jovian-type planets. All of these processes depend sensitively on either the total amount of gas or the ratio of the gas and dust mass. Dust masses can be estimated from the continuum flux of the disk, which is readily detectable at sub-millimeter ( $\mathrm{mm}$ ) wavelengths. However, the main gaseous component $\mathrm{H}_{2}$ does not have any strong emission lines that can trace the bulk of the disk mass, so that other tracers need to be used. Emission from the CO molecule and its isotopologues is commonly used as a mass tracer of molecular gas across astronomical environments (for reviews see, e.g. van Dishoeck \& Black 1987; Bolatto et al. 2013; Bergin \& Williams 2017). CO is resistant to photodissociation because it can selfshield against UV photons and is thus a molecule that can trace $\mathrm{H}_{2}$ in regions with low dust shielding (van Dishoeck \& Black 1988; Viala et al. 1988; Lee et al. 1996; Visser et al. 2009). $\mathrm{CO}$ also has, in contrast with $\mathrm{H}_{2}$, strong rotational lines, coming from states that can be populated at $20 \mathrm{~K}$, the freeze-out temperature of $\mathrm{CO}$. In most astronomical environments, $\mathrm{CO}$ is also chemically stable due to the large binding energy of the $\mathrm{C}-\mathrm{O}$ bond. This chemical stability means that $\mathrm{CO}$ is usually the second most abundant gas-phase molecule and the main volatile carbon reservoir in molecular astronomical environments. Thus, the recent finding that $\mathrm{CO}$ emission from protoplanetary disks is very weak came as a big surprise and implies that $\mathrm{CO}$ may be highly underabundant (Bruderer et al. 2012; Favre et al. 2013; Du et al. 2015; Kama et al. 2016; Ansdell et al. 2016). Is CO transformed to other species or are the majority of disks poor in gas overall? 
By extrapolating the chemical behaviour of $\mathrm{CO}$ from largescale astronomical environments to protoplanetary disks, it was expected that only two processes need to be accounted for in detail to determine the gaseous $\mathrm{CO}$ abundance throughout most of the disk: photodissociation and freeze-out of CO (Dutrey et al. 1997; van Zadelhoff et al. 2001, 2003). This was the outset for the results reported by Williams \& Best (2014) who computed a suite of disk models with parametrised chemical and temperature structures, to be used for the determination of disk gas masses from the computed line emission of CO isotopologues. This method was expanded by Miotello et al. $(2014,2016)$ who calculated the temperature, $\mathrm{CO}$ abundance and excitation selfconsistently using the thermo-chemical code DALI ${ }^{1}$ (Bruderer et al. 2012; Bruderer 2013). Miotello et al. (2016) used a simple gas-grain network that includes $\mathrm{CO}$ photodissociation, freezeout and grain-surface hydrogenation of simple species, but no full grain surface chemistry. DALI also computes the full 2D dust and gas temperature structure, important for determining the regions affected by freeze-out and emergent line emission. Because emission from the main $\mathrm{CO}$ isotopologue ${ }^{12} \mathrm{C}^{16} \mathrm{O}$ is often optically thick, most observations target the rarer $\mathrm{CO}$ isotopologues. These do not necessarily follow the highly abundant ${ }^{12} \mathrm{C}^{16} \mathrm{O}$ as ${ }^{12} \mathrm{C}^{16} \mathrm{O}$ can efficiently shield itself from photodissociating UV radiation at lower $\mathrm{H}_{2}$ column densities compared with the less abundant isotopologues. As such, the rarer isotopologues are dissociated over a larger region of the disk, an effect known as isotope-selective photodissociation (see, e.g. Visser et al. 2009). The combined effects of the different temperature structure and isotope-selective photodissociation change the emission strengths of the CO isotopologues by up to an order of magnitude compared with the predictions of Williams \& Best (2014).

When either of these model predictions including photodissociation and freeze-out are applied to ALMA observations of large samples of disks, still low gas masses are determined: inferred gas masses are close to, or lower than, the calculated dust mass from the same observations instead of the expected 100:1 ratio (Ansdell et al. 2016; Pascucci et al. 2016; Miotello et al. 2017; Long et al. 2017). While it is possible that these disks are indeed very gas depleted, independent determinations of the gas masses such as from far-infrared HD data (see, e.g. Bergin et al. 2013; McClure et al. 2016; Trapman et al. 2017) and mass accretion rates (Manara et al. 2016) imply that the $\mathrm{CO} / \mathrm{H}_{2}$ abundance ratio is likely much lower than expected, at least in the $\mathrm{CO}$ emitting part of the disk.

Multiple mechanisms have been proposed to explain this low $\mathrm{CO}$ abundance, both chemical and physical. A physical argument for the low $\mathrm{CO}$ abundances comes from the vertical mixing of the gas together with settling of dust. Kama et al. (2016) argued that the low CO abundance in the upper emitting layers of the outer disk can be explained by the constant vertical cycling of gaseous $\mathrm{CO}$. Every vertical cycle some $\mathrm{CO}$ will freeze-out onto grains that have grown and settled below the $\mathrm{CO}$ snow surface. These larger grains do not cycle back up again to the warmer regions where $\mathrm{CO}$ can be returned to the gas. They show that the $\mathrm{CO}$ abundance can be significantly lowered over the disk lifetime. This mechanism also predicts a strong anti-correlation between age and measured $\mathrm{CO}$ abundance. The mechanism can explain the destruction of $\mathrm{CO}$ in the warm layers, such as reported by Schwarz et al. (2016) and at the same time explain the lower than expected $\mathrm{H}_{2} \mathrm{O}$ abundances found in the outer disk of TW Hya and other disks by Hogerheijde et al. (2011) and Du et al. (2017). However, this mechanism cannot

\footnotetext{
1 http://www .mpe.mpg.de/ facchini/DALI/
}

explain the low abundance of $\mathrm{CO}$ inside of the $\mathrm{CO}$ iceline, the radial location of the snow surface at the midplane, as inferred by Zhang et al. (2017) for TW Hya.

Alternatively, there are various chemical mechanisms that destroy CO, sometimes referred to as "chemical depletion". Some of the proposed chemical pathways start with the destruction of gaseous $\mathrm{CO}$ by $\mathrm{He}^{+}$, leading to the formation and subsequent freeze-out of $\mathrm{CH}_{4}$ (Aikawa et al. 1999; Eistrup et al. 2016) or, when computed at slightly higher temperatures, the gas-phase formation of $\mathrm{C}_{2} \mathrm{H}_{2}$ and subsequent freeze-out and further chemical alteration on the grain-surface (Yu et al. 2016). Another pathway to destroy $\mathrm{CO}$ is the reaction with $\mathrm{OH}$ to form $\mathrm{CO}_{2}$, either in the gas phase (Aikawa et al. 1999), or on the grain-surface (Furuya \& Aikawa 2014; Reboussin et al. 2015; Drozdovskaya et al. 2016; Eistrup et al. 2016; Schwarz et al. 2018). The formation of $\mathrm{CO}_{2}$ through the grain-surface route seems to be most efficient at temperatures around $25 \mathrm{~K}$, just above the freeze-out temperature of $\mathrm{CO}$. A third pathway to destroy $\mathrm{CO}$ is the hydrogenation of $\mathrm{CO}$ on the dust grain-surface forming $\mathrm{CH}_{3} \mathrm{OH}$ (Cuppen et al. 2009; Yu et al. 2016; Eistrup et al. 2018). All of these models start with a high abundance of $\mathrm{CO}$ and modify the abundance through chemical processes. Alternatively, there are models that do not have CO initially as they assume that, due to some reset process, the gas is fully ionised or atomic at the start of the calculation (Eistrup et al. 2016; Molyarova et al. 2017). Due to the high abundance of $\mathrm{OH}$ during the transition of atomic to molecular gas, $\mathrm{CO}_{2}$ can be efficiently formed. At low temperatures $(<50 \mathrm{~K}) \mathrm{CO}_{2}$ becomes the most abundant carbon-bearing species.

All of these $\mathrm{CO}$ destruction processes are driven by dissociating or ionising radiation, either UV photons, X-rays or cosmic rays. In regions where UV photons and X-rays are not able to penetrate, cosmic rays drive the chemistry, so that the chemical timescales of $\mathrm{CO}$ processing are strongly dependent on the cosmic-ray ionisation rate (Reboussin et al. 2015; Eistrup et al. 2018). Indeed, Eistrup et al. (2016) show that chemical evolution during the disk lifetime in the dense midplane is negligible if the only source of ionisation is provided by the decay of radioactive nuclides. In line with these results, Schwarz et al. (2018) find that even in the warm molecular layers either a cosmic-ray ionisation rate of $10^{-17} \mathrm{~s}^{-1}$ or a strong X-ray field is needed to significantly destroy CO. High cosmic-ray ionisation rates are not expected if the proto-stellar magnetic field is sufficiently strong to deflect galactic cosmic rays (Cleeves et al. 2015).

The goal of this paper is to study the chemical pathways that can destroy $\mathrm{CO}$ in those regions of the disk that are sufficiently shielded from UV photons such as that near the disk midplane. The effectiveness and timescale of CO destruction pathways as functions of temperature, density and cosmic-ray ionisation rate are investigated for comparison with the increasing number of ALMA surveys of $\mathrm{CO}$ in disks in the 1-10 Myr age range. We also study the effect of the assumed grain-surface chemistry parameters, in particular the tunnelling barrier width and the diffusion-to-binding energy ratio. To be able to do this study in an as general sense as possible, we do not restrict ourselves to any specific disk structure but instead perform a parametric study of temperature, density and cosmic-ray ionisation rate over a range representative of a significant portion of the disk mass.

\section{Methods}

\subsection{Parameter space}

To constrain the amount of chemical processing of $\mathrm{CO}$ in shielded regions of disks, a grid of chemical and physical 
Table 1. Physical and chemical parameters explored.

\begin{tabular}{lccc}
\hline \hline Parameter & Symbol & Range & Fiducial value \\
\hline Temperature & $T$ & $10-40 \mathrm{~K}$ & - \\
Density & $n$ & $10^{6}-10^{12} \mathrm{~cm}^{-3}$ & - \\
Cosmic-ray ionisation rate & $\zeta_{\mathrm{H}_{2}}$ & $10^{-18}-10^{-16} \mathrm{~s}^{-1}$ & - \\
Tunnelling barrier width & $a_{\text {tunnel }}$ & $0.5-2.5 \AA$ & $1 \AA$ \\
Diffusion-to-binding energy ratio & $f_{\text {diff }}$ & $0.1-0.5$ & 0.3 \\
\hline
\end{tabular}

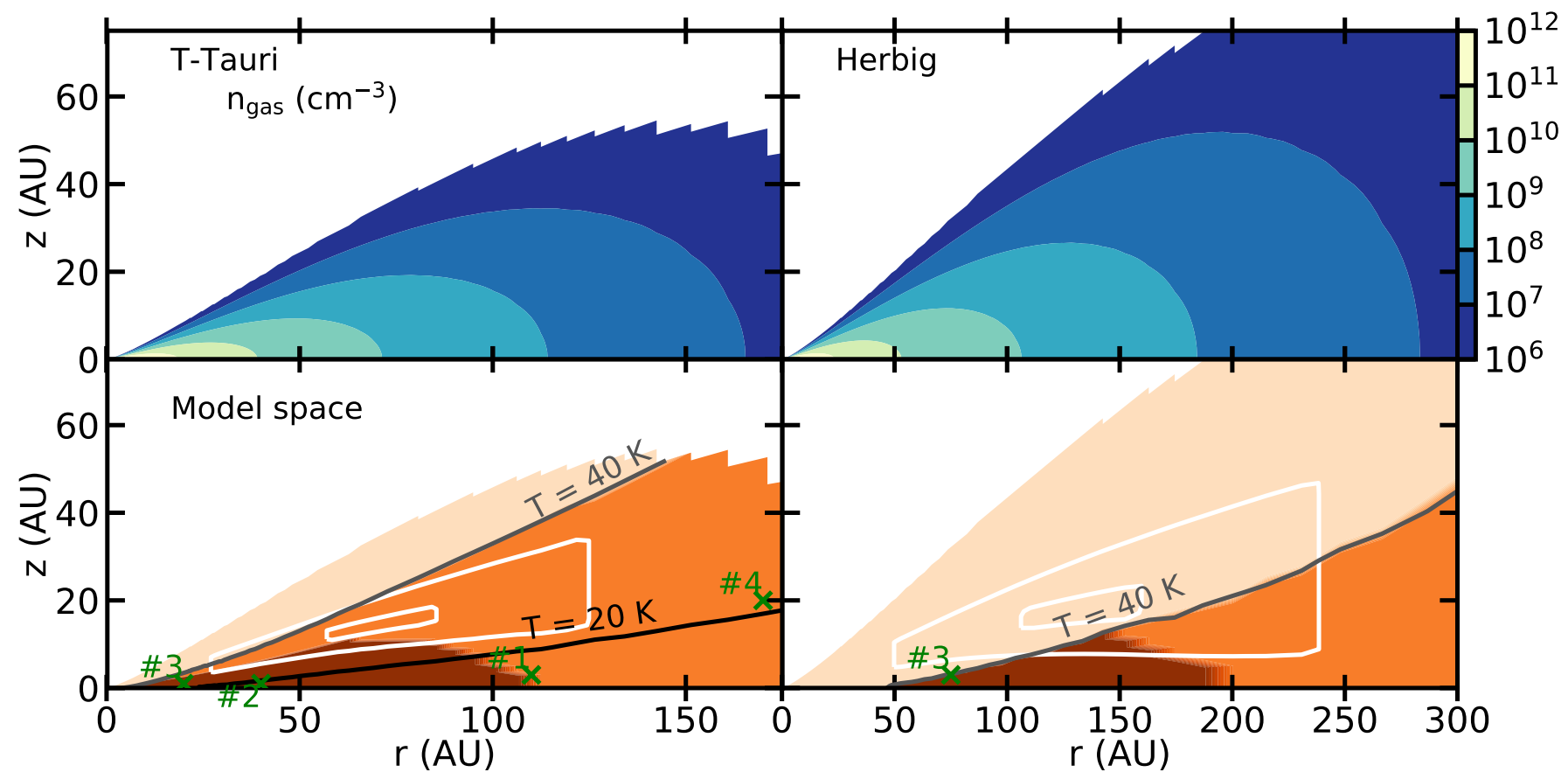

Fig. 1. Number density (top panels) and region of the disk included in the parameter study (bottom panels) for a typical $\mathrm{T}$ Tauri $\left(L=0.3 L_{\odot}\right.$; left panels) and Herbig ( $L=20 L_{\odot}$; right panels) disk model (see Appendix A for details). The part of the disk that is included in the physical models is shown in dark orange and is bound by the highest temperature included $(40 \mathrm{~K})$ and the restriction that the UV is fully shielded. Light orange denotes the region with temperatures and densities probed by our models but with some low level of UV. White contours show the regions contributing to $25 \%$ and $75 \%$ of the emission from the $\mathrm{C}^{18} \mathrm{O} 3-2$ line. The green crosses numbered \#1, \#2, \#3 and \#4 are approximate locations of the representative models of Sect. 3.1.3.

conditions typical for disk midplanes inside and outside the $\mathrm{CO}$ iceline is investigated. The explored parameter range is given in Table 1. The disk midplane is assumed to be shielded from stellar and interstellar UV photons. As such, cosmic-ray-induced photons are the only source of UV photons included in the model. The region is also assumed to be shielded from the most intense fluxes of stellar X-rays. The effects of moderate X-ray ionisation rates are similar to the effects of scaled-up cosmic-ray ionisation rates (Bruderer et al. 2009).

There are two steps in this parameter study. First a grid of chemical models for different physical conditions are computed. Temperatures, densities and cosmic-ray ionisation rates typical for cold, shielded regions of protoplanetary disks are used. For these models typical chemical parameters were used. In particular, the tunnelling barrier width $\left(a_{\text {tunnel }}\right)$ and diffusionto-binding energy ratio $\left(f_{\text {diff }}\right)$, characterizing surface chemistry (see Sect. 2.2), were kept constant at $1 \AA$ and 0.3 respectively.

For typical T Tauri and Herbig disks, the physical conditions probed by our models are shown in Fig. 1. The exact specifications of the models are presented in Appendix A. Both models assume a tapered power-law surface density distribution and a Gaussian vertical distribution for the gas. The dust and gas surface densities follow the same radial behaviour with an assumed global gas-to-dust mass ratio of 100 . Vertically there is more dust mass near the mid plane to simulate dust settling. The temperatures and densities that are included in the chemical models are shown in orange in the bottom panel in Fig. 1. The dark orange regions are those that are also completely shielded from VUV radiation. The gas is considered shielded if the intensity of external UV radiation at $100 \mathrm{~nm}$ is less than $10^{-4}$ times the intensity at that wavelength from the Draine ISRF (Draine 1978; Shen et al. 2004). Our models are however more broadly applicable to other parts of the disks due to dynamical mixing, as described below.

As the T Tauri disk model is colder (because of the less luminous star) and more compact compared to the Herbig disk model, there is more mass in the region of the disk probed by our models for that disk. The exact extent and location of the region probed by our chemical models strongly depends on the chosen parameters of the disk model. In the Herbig model, the temperature never drops below $20 \mathrm{~K}$, as such CO is not frozen out anywhere in that disk model. For selected points in the physical parameter space, an additional grid of chemical models is explored (see Sect. 3.2). 


\subsection{Chemical network}

The chemical network used in this work is based on the chemical model from Walsh et al. (2015), as also used in Eistrup et al. (2016, 2018). The "RATE12" network from the UMIST Database for Astrochemistry forms the basis of the gas-phase chemistry (McElroy et al. 2013) ${ }^{2}$. RATE12 includes gas-phase two-body reactions, photodissociation and photoionisation, direct cosmicray ionisation, and cosmic-ray-induced photodissociation and ionisation. Three-body reactions are not included as they are not expected to be important at the densities used in this work. Photo- and X-ray ionisation and dissociation reactions are included in the network but their contribution is negligible because we assume the disk midplane is well shielded from all external sources of X-ray and UV photons.

Freeze-out (adsorption) onto dust grains and sublimation (desorption) of molecules is included. Molecules can desorb either thermally or via cosmic-ray-induced photodesorption (Tielens \& Hagen 1982; Hasegawa et al. 1992; Walsh et al. 2010, 2012). Molecular binding energies as compiled for RATE12 (McElroy et al. 2013) are used, updated with the values recommended in the compliation by Penteado et al. (2017), except for $\mathrm{NH}, \mathrm{NH}_{2}, \mathrm{CH}, \mathrm{CH}_{2}$ and $\mathrm{CH}_{3}$. For $\mathrm{NH}$ and $\mathrm{NH}_{2}$, we calculate new estimates using the formalism proposed by Garrod \& Herbst (2006) and the binding energy for $\mathrm{NH}_{3}(3130 \mathrm{~K}$; Brown \& Bolina 2007). For the $\mathrm{CH}_{x}$ radicals, the binding energy is scaled by the number of hydrogen atoms with the $\mathrm{CH}_{4}$ binding energy of $1090 \mathrm{~K}$ as reference (He \& Vidali 2014). A list of all the binding energies used in this work is given in Table B.2. The binding energy used for $\mathrm{H}_{2}, 430 \mathrm{~K}$, predicts complete freeze-out of $\mathrm{H}_{2}$ at temperatures up to $15 \mathrm{~K}$ at densities of $10^{12} \mathrm{~cm}^{-3}$. However, at similar densities, $\mathrm{H}_{2}$ freezes-out completely at much lower temperatures (Cuppen \& Herbst 2007). The binding energy used here is the $\mathrm{H}_{2}$ to $\mathrm{CO}$ binding energy, whereas the $\mathrm{H}_{2}$ to $\mathrm{H}_{2}$ binding energy is expected to be much lower (Cuppen \& Herbst 2007). As such we modify the binding energy of $\mathrm{H}_{2}$ such that it is $430 \mathrm{~K}$ as long as there is less than one monolayer of $\mathrm{H}_{2}$ ice on the grain. Above two monolayers of $\mathrm{H}_{2}$ ice, we use the $\mathrm{H}_{2}$ on $\mathrm{H}_{2}$ binding energy of $100 \mathrm{~K}$. Between these two regimes, the binding energy of $\mathrm{H}_{2}$ is linearly dependent on the coverage of $\mathrm{H}_{2}$ ice. This is a different approach compared to that described in Hincelin et al. (2015) and Wakelam et al. (2016) but it has a similar effect on the $\mathrm{H}_{2}$ ice abundance. In all cases $E_{\text {diff }}=f_{\text {diff }} \times 430 \mathrm{~K}$ for the diffusion of $\mathrm{H}_{2}$.

Experimentally determined photodesorption yields are used where available (see, e.g., Öberg et al. 2009c,b,a), specifically $2.7 \times 10^{-3} \mathrm{CO}$ molecules per photon is used from Öberg et al. $(2009 \mathrm{c})$. We note that a large range of CO photodesorption yields, between $4 \times 10^{-4}$ and $0.25 \mathrm{CO}$ molecules per photon, are available in the literature due to the significant effects of experimental conditions (Öberg et al. 2007, 2009c; Muñoz Caro et al. 2010, 2016; Fayolle et al. 2011; Chen et al. 2014; Paardekooper et al. 2016). Fayolle et al. (2011) show that temperature and the wavelength of the incident photon strongly influence the photodesorption yield. For all species without experimentally determined photodesorption yields, a value of $10^{-3}$ molecules photon $^{-1}$ is used. The sticking efficiency is assumed to be 1 for all species except for the atomic hydrogen that leads to $\mathrm{H}_{2}$ formation.

The formation of $\mathrm{H}_{2}$ is implemented following Cazaux \& Tielens (2004) (see Appendix B.2 for a summary). This formalism forms $\mathrm{H}_{2}$ directly out of gas phase $\mathrm{H}$. This fraction of

\footnotetext{
2 http://www.udfa.net
}

atomic hydrogen is not available for reactions on the grain surface. About $50 \%$ of the atomic hydrogen is used to form $\mathrm{H}_{2}$ is this way. The remaining atomic hydrogen freezes-out on the grain surface and participates in the grain surface chemistry. Using this formalism ensures that the abundance of atomic $\mathrm{H}$ does not depend on the adopted grain-surface parameters. The balance between $\mathrm{H}_{2}$ formation and $\mathrm{H}_{2}$ destruction by cosmic rays produces an atomic $\mathrm{H}$ abundance in the gas that will always be around $1 \mathrm{~cm}^{-3}$ independent of the total $\mathrm{H}$ nuclei abundance.

For the grain-surface reactions, we use the reactions included in the Ohio State University (OSU) network ${ }^{3}$ (Garrod et al. 2008). The gas phase network is supplemented with reactions for important chemicals, for example the $\mathrm{CH}_{3} \mathrm{O}$ radical, that are not included in RATE 12. The destruction and formation reactions for these species are taken from the OSU network. The grain-surface network also includes additional routes to water formation as studied by Cuppen et al. (2010) and Lamberts et al. (2013). The grain-surface reactions are calculated assuming the LangmuirHinshelwood mechanism. Only the top two layers of the ice are chemically "active" and we assume that the chemically active layers have the same composition as the bulk ice. No reactiondiffusion competition for grain-surface reactions with a reaction barrier is included (Garrod \& Pauly 2011). The exact equations used to calculate the rates can be found in Appendix B.3.

The rates for the grain-surface reactions greatly depend on two quantities, the tunnelling barrier $\left(a_{\text {tunnel }}\right)$ and the diffusionto-binding energy ratio $\left(f_{\text {diff }}\right)$. $a_{\text {tunnel }}$ is usually taken to lie between 1 and $1.5 \AA$ (Garrod \& Pauly 2011; Walsh et al. 2015; Eistrup et al. 2016), and we test the range between 0.5 and $2.5 \AA$. The diffusion-to-binding energy ratio is generally taken to range between 0.3 and 0.5 (Walsh et al. 2015; Cuppen et al. 2017), although recent quantum chemical calculations predict values as low as $f_{\text {diff }}=0.15$ for $\mathrm{H}$ on crystalline water ice (Senevirathne et al. 2017). On the other hand, recent experiments suggest fast diffusion rates for $\mathrm{CO}$ on $\mathrm{CO}_{2}$ and $\mathrm{H}_{2} \mathrm{O}$ ices (Lauck et al. 2015; Cooke et al. 2018). The range tested here, $f_{\text {diff }}=0.1$ to $f_{\text {diff }}=0.5$, encompasses this measured range.

The chemical models are initialised with molecular abundances. The full list of abundances is given in Appendix B.1. Fully atomic initial conditions are not investigated.

\subsection{CO destruction routes}

There are three main pathways to destroy $\mathrm{CO}$ (see Sect. 1):

1. $\mathrm{sCO}+\mathrm{sH} \longrightarrow \mathrm{sHCO}$, leading to $\mathrm{sCH}_{3} \mathrm{OH}$

2. $\mathrm{sCO}+\mathrm{sOH} \longrightarrow \mathrm{sCO}_{2}+\mathrm{sH}$

3. $\mathrm{CO}+\mathrm{He}^{+} \longrightarrow \mathrm{C}^{+}+\mathrm{O}+\mathrm{He}$, leading to $\mathrm{CH}_{4}$ and $\mathrm{C}_{2} \mathrm{H}_{6}$ where $\mathrm{sX}$ denotes that species $\mathrm{X}$ is on the grain-surface. The interactions of these reactions with each other and the major competing reactions are shown in Fig. 2. For each of these reactions, a short analysis on the resulting rates is presented to explain the behaviour of the $\mathrm{CO}$ abundance as shown in Sect. 3 using the rate coefficients derived in Appendix B.3. Table 2 gives an overview of the symbols used, whereas Table 3 shows the sensivity to assumed parameters.

The reaction:

$\mathrm{sCO}+\mathrm{sH} \longrightarrow \mathrm{sHCO}$

happens on the surface and through further hydrogenation of sHCO leads to the formation of $\mathrm{sCH}_{3} \mathrm{OH}$. The initial step in this process is the most important and rate-limiting step. This reaction has a barrier, $E_{\mathrm{bar}}=2500 \mathrm{~K}$ (Woon 2002), which makes

http://faculty.virginia.edu/ericherb/research.html 


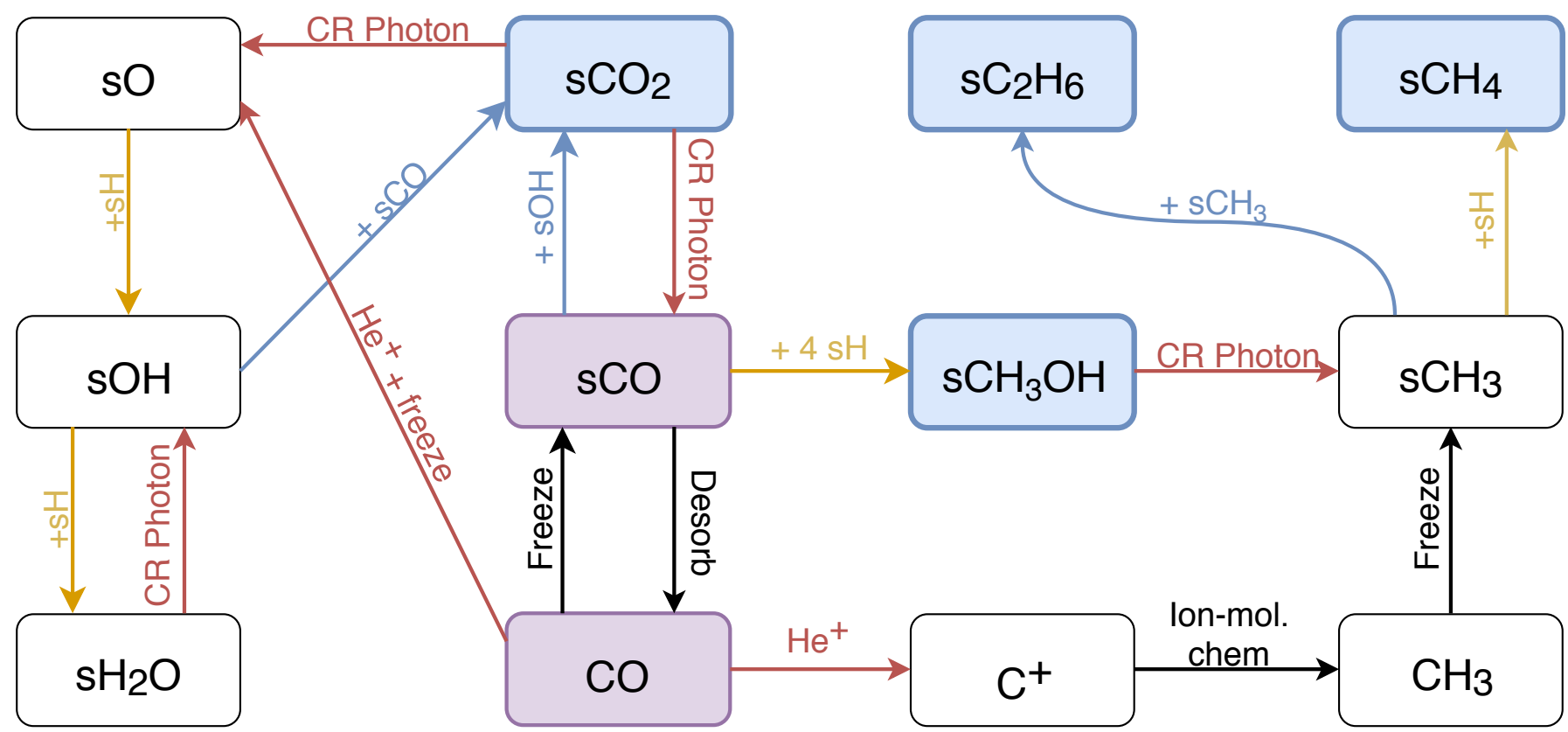

Fig. 2. Chemical reaction network showing the major $\mathrm{CO}$ destruction pathways and important competing reactions. Red arrows show reactions that are mediated directly or indirectly by cosmic-ray photons, yellow arrows show hydrogenation reactions and blue arrows show grain-surface reactions. The initial major carbon carrier $(\mathrm{CO})$ is shown in grey-purple. Stable products of $\mathrm{CO}$ processing are denoted with blue boxes. sX denotes that species $\mathrm{X}$ is on the grain surface.

Table 2. Symbol overview for the rate equations.

\begin{tabular}{ccc}
\hline \hline Symbol & Parameter & Value [Unit] \\
\hline$R_{\mathrm{X}+\mathrm{Y}}$ & X + Y reaction rate & {$\left[\mathrm{cm}^{-3} \mathrm{~s}^{-1}\right]$} \\
$n_{\mathrm{X}, \mathrm{A}}$ & Number density of species X in phase A & {$\left[\mathrm{cm}^{-3}\right]$} \\
$N_{\text {sites }}$ & Number of molecules per layers per grain & $10^{6}$ \\
$n_{\text {grain }}$ & Number density of grains & $2.2 \times 10^{-12} n_{\mathrm{gas}}\left[\mathrm{cm}^{-3}\right]$ \\
$C_{\text {grain }}$ & Ice mantle dependent prefactor & $\min \left[1,\left(\frac{N_{\mathrm{act}}^{2} N_{\text {sites }}^{2}}{n_{\text {grain }}^{2}}\right)\right] /\left(N_{\text {sites }} n_{\text {grain }}\right)\left[\mathrm{cm}^{3}\right]$ \\
$\mu$ & Reduced mass of the reacting species & {$[\mathrm{g}]$} \\
$E_{\text {bar }}$ & Height of the reaction barrier & {$[\mathrm{erg}]$} \\
$E_{\text {bind,X }}$ & Binding energy of species X & {$[\mathrm{erg}]$} \\
$v_{\mathrm{X}}$ & Vibration frequency of species X & Eq. (B.4) $\left[\mathrm{s}^{-1}\right]$ \\
\hline
\end{tabular}

tunnelling of $\mathrm{H}$ very important. The total $\mathrm{CO}$ destruction rate, assuming that tunnelling dominates for the reaction barrier, and that the thermal rate dominates for $\mathrm{H}$ hopping, can be given by

$$
\begin{aligned}
R_{\mathrm{CO}+\mathrm{H}} & =\frac{n_{\mathrm{CO}, \text { ice }} n_{\mathrm{H}, \text { ice }}}{n_{\mathrm{CO}, \text { total }}} C_{\text {grain }} \exp \left[-\frac{2 a_{\text {tunnel }}}{\hbar} \sqrt{2 \mu E_{\mathrm{bar}}}\right] \\
& \times\left(v_{\mathrm{H}} \exp \left[-\frac{f_{\text {diff }} E_{\text {bind, }}}{k T}\right]+v_{\mathrm{CO}} \exp \left[-\frac{f_{\text {diff }} E_{\text {bind,CO }}}{k T}\right]\right) .
\end{aligned}
$$

As noted above, the tunnelling barrier, $a_{\text {tunnel }}$, is the most important parameter for determining the rate. Changing $a_{\text {tunnel }}=0.5 \AA$ to $a_{\text {tunnel }}=1.5 \AA$ decreases the destruction rate through hydrogenation by eight orders of magnitude. This reaction is also suppressed in regions of high temperature where $n_{\mathrm{CO} \text {,ice }} / n_{\mathrm{CO} \text {,total }}$ is low.

The amount of $\mathrm{H}$ in the ice is set by the balance of freezeout of $\mathrm{H}$ and the reaction speed of $\mathrm{H}$ with species in the ice. Desorption of $\mathrm{H}$ is negligible compared with the reaction of $\mathrm{H}$ with radicals on the grain in most of the physical parameter space explored. As such there is no strong decrease in the rate near the $\mathrm{H}$ desorption temperature. This also means that the competition of other iceborn radicals for reactions with $\mathrm{H}$ strongly influences the rates.

At the lowest temperatures, the rates are also slightly suppressed as the hopping rate is slowed. The rate is maximal around the traditional $\mathrm{CO}$ iceline temperature of around $20 \mathrm{~K}$ as $n_{\mathrm{CO} \text {,ice }} / n_{\mathrm{CO} \text {,total }}$ is still high, while thermal hopping is efficient. This is especially so for low values of $f_{\text {diff }}$, increasing the hopping and thus the reaction rate. This reaction does not strongly depend on density since the absolute flux of $\mathrm{H}$ arriving on grains is nearly constant as function of total gas density and the rest of the rate only depends on the fraction of $\mathrm{CO}$ that is frozen out, not the total amount.

The second reaction is the formation of $\mathrm{CO}_{2}$ through the grain-surface reaction:

$\mathrm{sCO}+\mathrm{sOH} \longrightarrow \mathrm{sCO}_{2}+\mathrm{sH}$,

which has a slight barrier of $400 \mathrm{~K}$ (Arasa et al. 2013). It competes with the reaction $\mathrm{sCO}+\mathrm{sOH} \longrightarrow \mathrm{sHOCO}$. We assume that most of the HOCO formed in this way will be converted into 
Table 3. Chemical trends with variations in parameters.

\begin{tabular}{llcc}
\hline \hline Parameter & & $\mathrm{sCO}+\mathrm{sOH}$ & $\mathrm{sCO}+\mathrm{sH}$ \\
\hline$\uparrow n$ & $\downarrow x_{\mathrm{H}}$ & $\uparrow$ & $\downarrow$ \\
$\uparrow a_{\text {tunnel }}$ & $\downarrow P_{\text {reac }}(\mathrm{sCO}, \mathrm{sH})$ & - & $\downarrow$ \\
$\uparrow a_{\text {tunnel }}$ & $\left.\downarrow P_{\text {reac }}(\mathrm{sOH}, \mathrm{sH})_{2}\right)$ & $\uparrow$ & - \\
$\uparrow f_{\text {diff }}$ & $\downarrow \mathrm{CO}$ mobility & $\downarrow$ & - \\
\hline
\end{tabular}

$\mathrm{CO}_{2}$ as seen in the experiments (Watanabe et al. 2007; Oba et al. 2010; Ioppolo et al. 2013) and that is also required to explain $\mathrm{CO}_{2}$ ice observations. As such we suppress the explicit $\mathrm{HOCO}$ formation channel in our model.

The reaction rate for Eq. (3) is given by

$$
\begin{aligned}
R_{\mathrm{CO}+\mathrm{OH}} & =\frac{n_{\mathrm{CO}, \text { ice }} n_{\mathrm{OH}, \text { ice }}}{n_{\mathrm{CO}, \text { total }}} C_{\text {grain }} \exp \left[-\frac{E_{\mathrm{bar}}}{k T}\right] \\
& \times\left(v_{\mathrm{OH}} \exp \left[-\frac{f_{\text {diff }} E_{\mathrm{bind}, \mathrm{OH}}}{k T}\right]+v_{\mathrm{CO}} \exp \left[-\frac{f_{\text {diff }} E_{\text {bind,CO }}}{k T}\right]\right) .
\end{aligned}
$$

As $\mathrm{OH}$ has a high binding energy of $2980 \mathrm{~K}$ (He \& Vidali 2014), sublimation of $\mathrm{OH}$ can be neglected. $\mathrm{CO}$ sublimation is still important even though the rate is again not dependent on the total CO abundance. Due to the strong temperature dependence of the reaction barrier and the $\mathrm{CO}$ hopping rate, this rate is maximal at temperatures just above the $\mathrm{CO}$ desorption temperature. Finally, the reaction rate depends on the $\mathrm{OH}$ abundance. $\mathrm{OH}$ in these circumstances is generally created by the cosmicray-induced photodissociation of $\mathrm{H}_{2} \mathrm{O}$, which means that $\mathrm{CO}_{2}$ formation is fastest when there is a large body of $\mathrm{H}_{2} \mathrm{O}$ ice ${ }^{4}$. At late times $\mathrm{H}_{2} \mathrm{O}$ can also become depleted, with $\mathrm{CO}_{2}$ being the major oxygen reservoir, lowering the supply of $\mathrm{OH}$ at late times. This lowers the $\mathrm{CO}_{2}$ production rate, and the destruction of $\mathrm{CO}_{2}$ can increase the $\mathrm{CO}$ abundance.

$\mathrm{CO}$ has competition with several other radicals for the reaction with $\mathrm{OH}$. The most important of these is the competition with $\mathrm{H}$. At low densities, the $x_{\mathrm{H}} / x_{\mathrm{CO}}$ is high, so $\mathrm{OH}$ will mostly react with $\mathrm{H}$ to reform $\mathrm{H}_{2} \mathrm{O}$. Similarly, when $\mathrm{H}$ mobility is increased, by assuming very narrow tunnelling barriers, $\mathrm{sCO}_{2}$ formation will slow down. At high density, $x_{\mathrm{H}} / x_{\mathrm{CO}}$ is low, and thus the competition for $\mathrm{OH}$ is won by $\mathrm{CO}$.

The last reaction is the only gas phase route:

$\mathrm{CO}+\mathrm{He}^{+} \longrightarrow \mathrm{C}^{+}+\mathrm{O}+\mathrm{He}$.

This reaction is limited by the ionisation rate of $\mathrm{He}$ and the subsequent competition for collisions of $\mathrm{He}^{+}$with abundant gas phase species. As such, the $\mathrm{CO}$ destruction rate can be expressed as

$R_{\mathrm{CO}+\mathrm{He}^{+}}=0.65 \zeta_{\mathrm{H}_{2}} \frac{x_{\mathrm{He}}}{x_{\mathrm{CO}}} \frac{k_{\text {ion,CO}} x_{\mathrm{CO}}}{\sum_{\mathrm{X}} k_{\mathrm{ion}, \mathrm{X}} x_{\mathrm{X}}}$

where $k_{\text {ion,X }}$ are the ion-neutral reaction rate coefficients for collisions between $\mathrm{He}^{+}$and the molecule and $x_{\mathrm{X}}$ is the abundance of species $X$. The abundances and rate coefficients for important alternative reaction partners of $\mathrm{He}^{+}$between 20 and $40 \mathrm{~K}$ are tabulated in Table 4, where we have summed the rate coefficients of reactions with multiple outcomes. At high abundances of $\mathrm{CO}$ and/or $\mathrm{N}_{2}$, the rate scales as

$R_{\mathrm{CO}+\mathrm{He}^{+}} \propto \frac{1}{x_{\mathrm{CO}}+x_{\mathrm{N}_{2}}}$,

4 Specifically, $\mathrm{H}_{2} \mathrm{O}$ is in the upper layers of the ice, but by construction our ice mantles are perfectly mixed.
Table 4. Rate coefficients for collisions with $\mathrm{He}^{+}$.

\begin{tabular}{lcc}
\hline \hline Reaction partner & Rate coefficients $\left(\mathrm{cm}^{-3} \mathrm{~s}^{-1}\right)$ & Gas abundance \\
\hline $\mathrm{H}_{2}$ & $1.14 \times 10^{-14}$ & 0.5 \\
$\mathrm{~N}_{2}$ & $1.6 \times 10^{-9}$ & $<2 \times 10^{-5}$ \\
$\mathrm{CO}$ & $1.6 \times 10^{-9}$ & $<10^{-4}$ \\
grains & $2.06 \times 10^{-4}$ & $2.2 \times 10^{-12}$ \\
\hline
\end{tabular}

which increases with lower abundances. If the sum of the gaseous abundances of $\mathrm{CO}$ and $\mathrm{N}_{2}$ is $<<3 \times 10^{-7}$, the rate becomes

$R_{\mathrm{CO}+\mathrm{He}^{+}}=0.65 \zeta_{\mathrm{H}_{2}} x_{\mathrm{He}} \frac{k_{\text {ion,CO }}}{k_{\text {ion, } \mathrm{H}_{2}} x_{\mathrm{H}_{2}}+k_{\text {ion,grains }} x_{\text {grains }}}$

which is independent of the $\mathrm{CO}$ abundance.

There are some assumptions in the model that will influence the rates of the chemical pathways discussed here. We do not expect the chemistry to be critically dependent on these assumptions but they might influence the chemical timescales and the relative importance of different chemical pathways. A few important assumptions and their effects on the chemistry are discussed in Appendix B.4.

\section{Results}

We have performed a parameter space study of the chemistry of $\mathrm{CO}$ under shielded conditions in protoplanetary disks. In this section we first present the results for the physical parameters studied, namely chemical evolution time, density, temperature and cosmic-ray ionisation rate. Figure 3 focuses on the effects of time and cosmic-ray ionisation rate, while Fig. 4 focuses on the effects of temperature and density. Together these figures show that the evolution of the $\mathrm{CO}$ abundance depends strongly on the physical conditions assumed in the chemical model, especially the temperature, in addition to the cosmic-ray ionisation rate identified earlier. Finally, the effects of the assumed chemical parameters on four positions in physical parameter space are studied.

\subsection{Physical parameter space}

\subsubsection{Importance of the cosmic-ray ionisation rate}

Consistent with previous studies, the cosmic-ray ionisation rate is found to be the driving force behind most of the changes in the $\mathrm{CO}$ abundance. A higher cosmic-ray ionisation rate allows the chemistry to evolve faster, but in a similar way. As such the cosmic-ray ionisation rate and chemical evolution time are mostly degenerate. Figure 3 presents an overview of the dependence of the total $\mathrm{CO}$ abundance (gas plus ice) on evolution time and $\zeta_{\mathrm{H}_{2}}$. CO can be efficiently destroyed in 1-3 Myr for $\zeta_{\mathrm{H}_{2}}>5 \times 10^{-18} \mathrm{~s}^{-1}$ and temperatures lower than $25 \mathrm{~K}$.

For models at $15 \mathrm{~K}$ and low densities of $10^{6} \mathrm{~cm}^{-3}$, the CO abundance behaviour does not show the degeneracy between $\zeta_{\mathrm{H}_{2}}$ and time. This is caused by the formation of NO in the ice. The NO abundance depends non-linearly on the cosmic-ray ionisation rate. A high abundance of NO in the ice lowers the abundance of available atomic $\mathrm{H}$ on the ice as it efficiently catalyses the formation of $\mathrm{H}_{2}$ on the ice. This effect has also been seen in Penteado et al. (2017) using the same network but under different conditions. A similar catalytic effect for the formation of $\mathrm{H}_{2}$ was first noted in the work by Tielens \& Hagen (1982). 


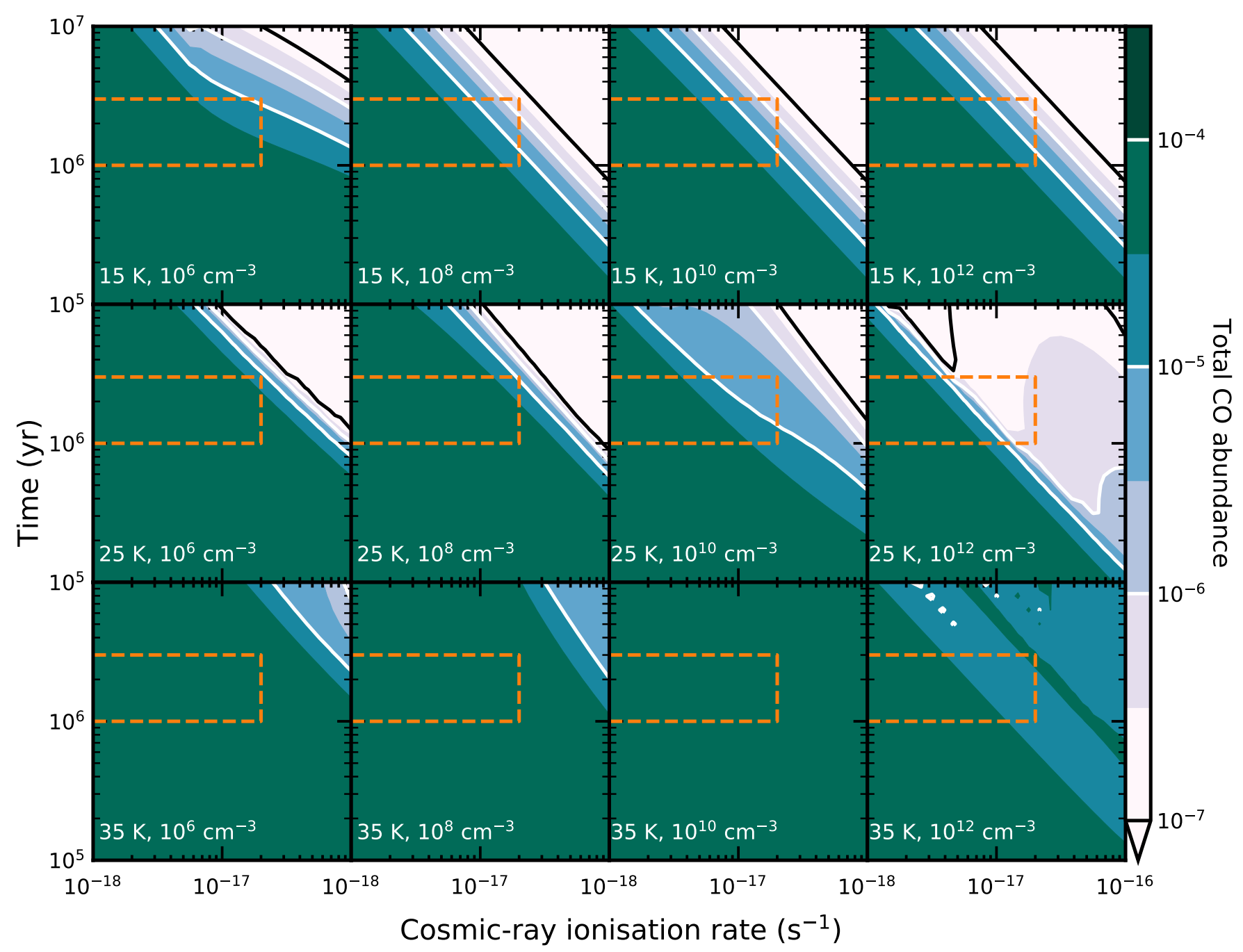

Fig. 3. Total CO abundance (gas and ice) as function of time and cosmic-ray ionisation rate for the fiducial chemical model. Each sub-figure has a different combination of temperature and density as denoted in the bottom left of each panel. Time and cosmic-ray ionisation rate are degenerate in most of the parameter space. The orange box denotes the combinations of $\zeta_{\mathrm{H}_{2}}$ and time most appropriate for protoplanetary disks.

At high density and temperature $\left(35 \mathrm{~K}, 10^{10} \mathrm{~cm}^{-3}\right)$, a sequence of $\mathrm{CO}$ destruction and then reformation is visible in the $\mathrm{CO}$ abundance. The first cycle of this was also seen and discussed in Eistrup et al. (2016) and can be attributed to the lower formation rate of $\mathrm{OH}$ due to a decrease in the $\mathrm{H}_{2} \mathrm{O}$ abundance on Myr timescales. Some models, especially those with a cosmicray ionisation rate of $10^{-16} \mathrm{~s}^{-1}$, can have five or more of these $\mathrm{CO}-\mathrm{CO}_{2}$ abundance inversions, while the $\mathrm{H}_{2} \mathrm{O}$ abundance continues to decrease. For the rest of the results in this section, a cosmic-ray ionisation rate of $10^{-17} \mathrm{~s}^{-1}$, is taken, thought to be typical for dense molecular clouds (e.g., Dalgarno 2006).

\subsubsection{Importance of temperature}

Figure 4 presents the total $\mathrm{CO}$ abundance over the entire densitytemperature grid at four time steps during the evolution of the chemistry. These figures demonstrate clearly that $\mathrm{CO}$ is efficiently destroyed only at low temperatures, $<30 \mathrm{~K}$. At early times, $\mathrm{CO}$ is most effectively destroyed at high densities and temperatures between 20 and $30 \mathrm{~K}$. In this range, the grain-surface formation of $\mathrm{CO}_{2}$ from the reaction between $\mathrm{CO}$ and $\mathrm{OH}$ is efficient. At these temperatures, $\mathrm{CO}$ is primarily in the gas phase but a small fraction resides on the grain surface where it is highly mobile. $\mathrm{OH}$ is created during the destruction of $\mathrm{H}_{2} \mathrm{O}$ ice. This reaction is most efficient under high-density conditions because atomic $\mathrm{H}$ competes with $\mathrm{CO}$ for reaction with the $\mathrm{OH}$ radical on the grain. At low densities, the relative abundance of $\mathrm{H}$ is higher in the models, thus greatly suppressing the formation rate of $\mathrm{CO}_{2}$ from $\mathrm{CO}+\mathrm{OH}$ on the grain.

At $3 \mathrm{Myr} \mathrm{CO}$ destruction below $20 \mathrm{~K}$ becomes visible. The total $\mathrm{CO}$ abundance in this temperature range is only weakly dependent on the density: $\mathrm{CO}$ destruction is efficient below $16 \mathrm{~K}$ at the lowest densities, while it is efficient up to $19 \mathrm{~K}$ at the highest densities. This range is strongly correlated with the fraction of $\mathrm{CO}$ residing in the ice.

At late times, $>5 \mathrm{Myr}$, there is a strong additional $\mathrm{CO}$ destruction at densities $<10^{9} \mathrm{~cm}^{-3}$ and at temperatures between 20 and $25 \mathrm{~K}$. At this point, the $\mathrm{C}^{+}$formed from the $\mathrm{CO}+\mathrm{He}^{+}$ reaction can efficiently be converted into $\mathrm{CH}_{4}$, which freezes out on the grains.

\subsubsection{Representative models}

Four points in the physical parameter space have been chosen for further examination. They are chosen such that they span the range of parameters that can lead to low total $\mathrm{CO}$ abundances within $10 \mathrm{Myr}$ and such that they sample different $\mathrm{CO}$ destruction routes. These points are given in Table 5 and are marked in 


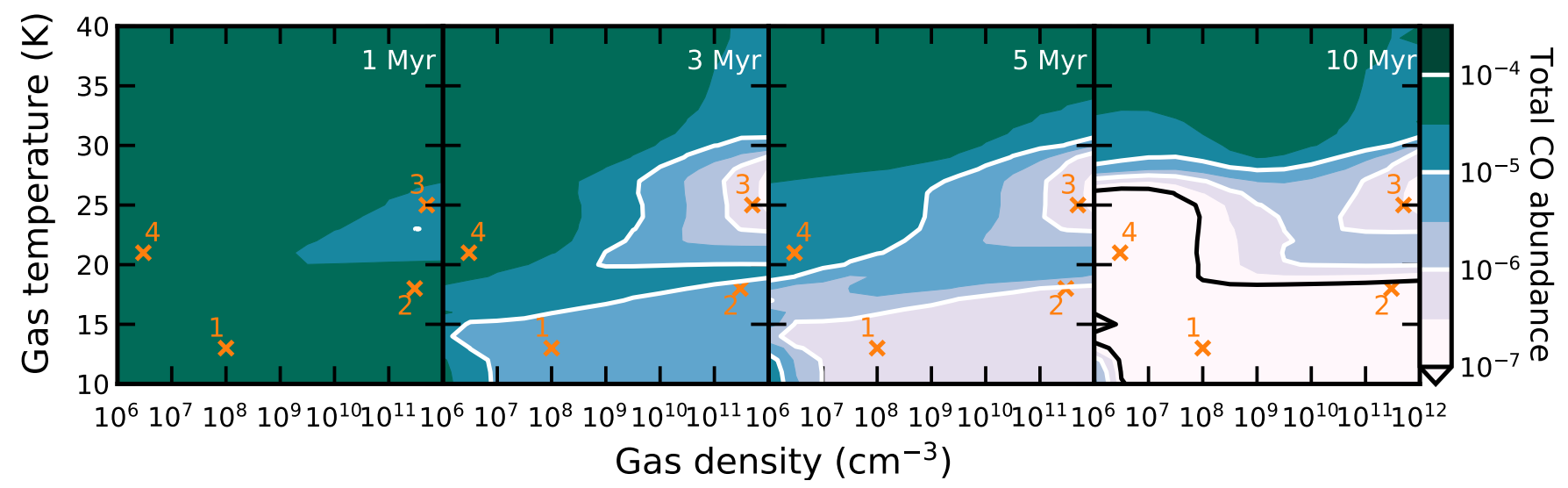

Fig. 4. Time evolution of $\mathrm{CO}$ abundance as function of gas temperature and density. Chemical evolution time is denoted in the upper right corner of each panel and $\zeta=10^{-17} \mathrm{~s}^{-1}$ for all of these models. The orange numbers show the locations of the physical conditions taken in Sect. 3.2.

Fig. 4. Figure 5 presents the total abundance (gas and ice) of $\mathrm{CO}$ and its stable reaction products as a function of time for the four physical conditions that have been chosen.

Models \#1 (13 K, $\left.10^{8} \mathrm{~cm}^{-3}\right)$ and \#2 $\left(18 \mathrm{~K}, 3 \times 10^{11} \mathrm{~cm}^{-3}\right)$ show very similar behaviours, even though they have very different densities. This is due to the combination of the active destruction pathway, $\mathrm{CO}$ hydrogenation, and the $\mathrm{H}_{2}$ formation prescription, which forces a constant atomic hydrogen concentration, leading to a constant $\mathrm{CO}$ destruction rate as a function of density. In both models, $90 \%$ of the $\mathrm{CO}$ has been converted into $\mathrm{CH}_{3} \mathrm{OH}$ in $2 \mathrm{Myr}$. Before this time the $\mathrm{H}_{2} \mathrm{CO}$ abundance is constant, balanced between the formation due to $\mathrm{CO}$ hydrogenation and destruction due to hydrogenation. As the $\mathrm{CO}$ abundance drops, and thus the formation rate of $\mathrm{H}_{2} \mathrm{CO}$ falls, so does its abundance. After slightly more than $2 \mathrm{Myr}$, methanol has reached a peak abundance close to $10^{-4}$. This marks the end of hydrogenation-driven chemical evolution as most molecules on the ice cannot be hydrogenated further. After this, cosmicray-induced dissociation dominates the abundance evolution, slowly destroying $\mathrm{CH}_{3} \mathrm{OH}$, forming $\mathrm{CH}_{4}$ and $\mathrm{H}_{2} \mathrm{O}$ and destroying $\mathrm{CO}_{2}$ forming $\mathrm{CO}$ and $\mathrm{O}$, both of which quickly hydrogenate to $\mathrm{CH}_{3} \mathrm{OH}$ and $\mathrm{H}_{2} \mathrm{O}$. A small amount of $\mathrm{CH}_{4}$ is further converted into $\mathrm{C}_{2} \mathrm{H}_{6}$, which happens more efficiently at higher densities, due to the lower availability of atomic $\mathrm{H}$.

The abundance traces for model \#3 $\left(25 \mathrm{~K}, 5 \times 10^{11} \mathrm{~cm}^{-3}\right)$ show two different destruction pathways for $\mathrm{CO}$ at a temperature where most of the $\mathrm{CO}$ is in the gas phase. The rise in $\mathrm{CO}_{2}$ abundance indicates that a significant portion of the $\mathrm{CO}$ reacts with $\mathrm{OH}$ on the grain-surface to form $\mathrm{CO}_{2}$. At $2 \mathrm{Myr}$, nearly $99 \%$ of the initial CO has been destroyed. Most of the CO has been incorporated into $\mathrm{CO}_{2}$ with a significant amount of carbon locked into $\mathrm{CH}_{4} \cdot \mathrm{CH}_{4}$ is formed from a sequence of reactions that begin with the destruction of $\mathrm{CO}$ by $\mathrm{He}^{+}$. At these late times, $\mathrm{C}_{2} \mathrm{H}_{6}$ acts as a carbon sink, slowly locking up carbon that is created in the form of the $\mathrm{CH}_{3}$ radical from the dissociation of $\mathrm{CH}_{4}$.

The abundance traces for model \#4 $\left(21 \mathrm{~K}, 3 \times 10^{6} \mathrm{~cm}^{-3}\right)$ are an outlier in this comparison. Most of the $\mathrm{CO}$ is in the gas phase at this temperature and density. It takes at least $2 \mathrm{Myr}$ to destroy $50 \%$ of the $\mathrm{CO}$ and $5 \mathrm{Myr}$ to destroy $90 \%$ of the CO. For the conditions shown here, most of the $\mathrm{CO}$ is destroyed in the gas phase by dissociative electron transfer with $\mathrm{He}^{+}$. This leads to the formation of hydrocarbons in the gas phase, primarily $\mathrm{CH}_{3}$ and $\mathrm{C}_{2} \mathrm{H}_{2}$, which freeze-out and are hydrogenated on the grain to form $\mathrm{CH}_{4}$ and $\mathrm{C}_{2} \mathrm{H}_{6}$ (as also seen by Aikawa et al. 1999).
Table 5. Physical parameters for the chemical parameters test.

\begin{tabular}{cccc}
\hline \hline Model \# & $n_{\text {gas }}\left(\mathrm{cm}^{-3}\right)$ & $T_{\text {gas }}(\mathrm{K})$ & $\zeta_{\mathrm{H}_{2}}\left(\mathrm{~s}^{-1}\right)$ \\
\hline 1 & $1 \times 10^{8}$ & 13 & $10^{-17}$ \\
2 & $3 \times 10^{11}$ & 18 & $10^{-17}$ \\
3 & $5 \times 10^{11}$ & 25 & $10^{-17}$ \\
4 & $3 \times 10^{6}$ & 21 & $10^{-17}$ \\
\hline
\end{tabular}

\subsection{Chemical parameter space}

For the four different cases listed in Table 5, a set of models with varying $a_{\text {tunnel }}$ and $f_{\text {diff }}$ have been computed. $a_{\text {tunnel }}$ primarily changes the reaction probability for grain-surface reactions involving atomic or molecular hydrogen that have a barrier, such as $\mathrm{sCO}+\mathrm{sH}$ and $\mathrm{sOH}+\mathrm{sH}_{2}$. The value of $f_{\text {diff }}$ changes the speed at which species can move over the grain-surface. Models \#1 and \#2 are both in the region of parameter space where $\mathrm{CO}$ is frozen out and thus sample pure grain-surface chemistry at low temperature and density, and at a slightly higher temperature and high density, respectively. Model \#3 is near the local minimum in gaseous CO abundance seen in Fig 4. Model \#4 is located in a region of parameter space where most changes are still ongoing at later times. Together these four cases should sample the different dominant $\mathrm{CO}$ destruction pathways.

The first row of Fig. 6 shows the total $\mathrm{CO}$ abundance as function of chemical parameters for different times for point \#1 in our physical parameter space with $\mathrm{CO}$ mostly in the ice. There is a strong dependence on the tunnelling barrier width $\left(a_{\text {tunnel }}\right)$. This is because the $\mathrm{CO}$ destruction in this temperature regime is dominated by the formation of $\mathrm{sHCO}$. The $\mathrm{sCO}+\mathrm{sH}$ reaction has a barrier which strongly limits this reaction, $\mathrm{H}$ tunnelling through this barrier thus increases the rate of $\mathrm{CO}$ destruction. Our fiducial value for the barrier width, $1 \AA$, is in the transition between slow hydrogenation at higher barrier widths and runaway hydrogenation at lower barrier widths. As such a $10 \%$ change to the barrier width around $1 \AA$ changes the abundance of $\mathrm{CO}$ by a factor of $\sim 3$.

There are only very weak dependencies on the diffusionto-binding energy ratio ( $\left.f_{\text {diff }}=E_{\text {diff }} / E_{\text {bind }}\right)$ for these very low temperatures. This points at a $\mathrm{CO}$ destruction process that is entirely restricted by the tunnelling efficiency of $\mathrm{H}$. If the $\mathrm{sCO}+$ $\mathrm{sH}$ reaction is quenched by a large barrier, $\mathrm{CO}$ destruction is so slow that, due to the destruction of $\mathrm{CO}_{2}$ by cosmic-ray-induced photons, the $\mathrm{CO}$ abundance is actually increased from the initial 


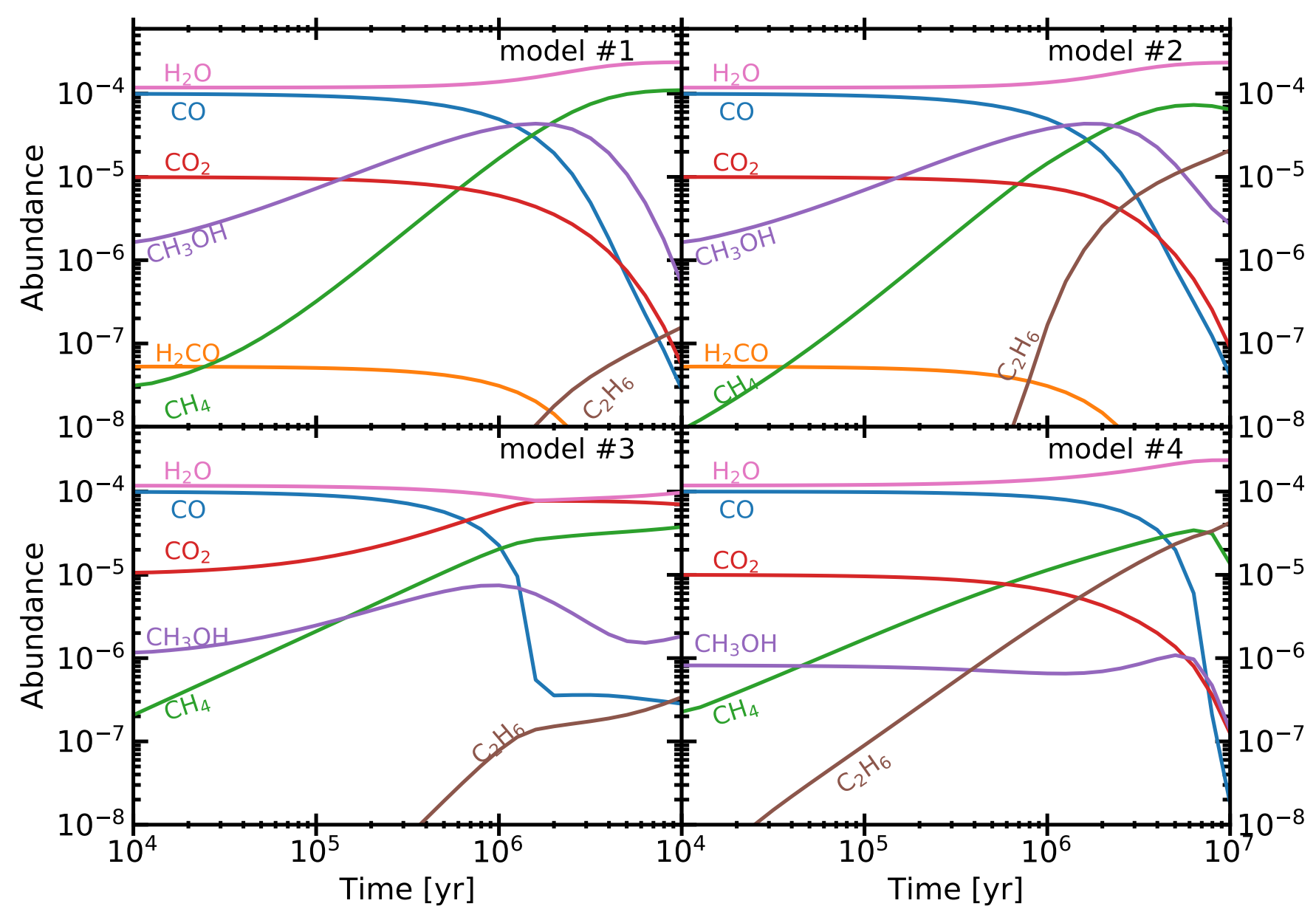

Fig. 5. Abundance traces of $\mathrm{CO}$ and its destruction products for the four points denoted in Fig. 4 with conditions as tabulated in Table 5. Plotted abundances are the sum of the gas and ice abundance for each species.

value. This happens at barrier widths larger than $1.5 \AA$. At the lowest $f_{\text {diff }}, \mathrm{CO}$ is turned into $\mathrm{CO}_{2}$ through $\mathrm{sCO}+\mathrm{sOH}$ at early times. At later times, the $\mathrm{CO}_{2}$ is destroyed, again by cosmic-rayinduced photons and more $\mathrm{CO}$ is formed, leading to a slower $\mathrm{CO}$ abundance decrease at late times.

The second row of Fig. 6 shows the total $\mathrm{CO}$ abundance as a function of chemical parameters for different times for point \#2. Since CO is frozen out for both case \#1 $(T=13 \mathrm{~K})$ and \#2 $(T=18 \mathrm{~K})$, there are strong similarities between the first and second row of models in Fig. 6. The only significant difference can be seen at $a_{\text {tunnel }}>1.0 \AA$ and $f_{\text {diff }}<0.2$. With these chemical parameters and these high densities $\left(3 \times 10^{11} \mathrm{~cm}^{-3}\right), \mathrm{sCO}+$ $\mathrm{sOH}$ can be an effective destruction pathway.

The third row of Fig. 6 shows the total $\mathrm{CO}$ abundance for point \#3 in our physical parameter space at $T=25 \mathrm{~K}$ and $n=5 \times 10^{11} \mathrm{~cm}^{-3}$. The large and irregular variation in CO abundance in this figure points at a number of competing processes. The two main processes destroying $\mathrm{CO}$ at this temperature and density are again $\mathrm{sCO}+\mathrm{sH}$ and $\mathrm{sCO}+\mathrm{sOH}$. These grain-surface reactions dominate the $\mathrm{CO}$ destruction even though $\mathrm{CO}$ is primarily in the gas phase. The fraction of time that $\mathrm{CO}$ spends on the grain is long enough to allow the aforementioned reactions to be efficient. At the smallest barrier widths $(<0.7 \AA)$, the conversion of $\mathrm{CO}$ into $\mathrm{CH}_{3} \mathrm{OH}$ through hydrogenation dominates the $\mathrm{CO}$ abundance evolution, but this pathway quickly gets inefficient if the tunnelling barrier is made wider. In the rest of the parameter space, the formation of $\mathrm{CO}_{2}$ dominates the abundance evolution.
Although CO is destroyed significantly in this model from 2 Myr onward, there is a clear region in parameter space where the $\mathrm{CO}$ destruction is slower. This region at high $f_{\text {diff }}$ and intermediate $a_{\text {tunnel }}$ has up to two orders of magnitude higher CO abundance compared with the rest of parameter space. The high $f_{\text {diff }}$ significantly slows down all reactions that are unaffected by tunnelling. In this region of parameter space, $\mathrm{CO}$ mobility is significantly lower than $\mathrm{H}$ mobility due to the latter being able to tunnel. This suppresses the $\mathrm{sCO}+\mathrm{sOH}$ route. On the other hand, the barrier is still too wide to allow efficient hydrogenation of $\mathrm{CO}$. With both main destruction routes suppressed in this region, it takes longer to reach a significant amount of $\mathrm{CO}$ destruction. Further increasing $a_{\text {tunnel }}$ from this point slows down the tunnelling of $\mathrm{H}$ and thus the formation rate of $\mathrm{H}_{2} \mathrm{O}$. This leads into a larger $\mathrm{OH}$ abundance on the ice, and thus a larger $\mathrm{CO}_{2}$ formation rate.

The fourth row of Fig. 6 shows the total $\mathrm{CO}$ abundance at different times for point \#4 at a low density of $3 \times 10^{6} \mathrm{~cm}^{-3}$ and $T=21 \mathrm{~K}$. At early times there is no strong destruction of $\mathrm{CO}$, after $3 \mathrm{Myr}$ there is only a small region where the abundance has dropped by a factor of three. $\mathrm{CH}_{3} \mathrm{OH}$ and $\mathrm{CO}_{2}$ are formed in this region of parameter space. At $5 \mathrm{Myr}$, there is a region at low barrier width, around $0.6 \AA$ and $f_{\text {diff }}>0.3$, where the hydrogenation of $\mathrm{CO}$ has led to a large decrease in the $\mathrm{CO}$ abundance. At $7 \mathrm{Myr}$ this process has caused a decrease of $\mathrm{CO}$ abundance of at least two orders of magnitude in this region. At the same time, the CO abundance over almost all of the parameter space has dropped by an order of magnitude. This is the effect 


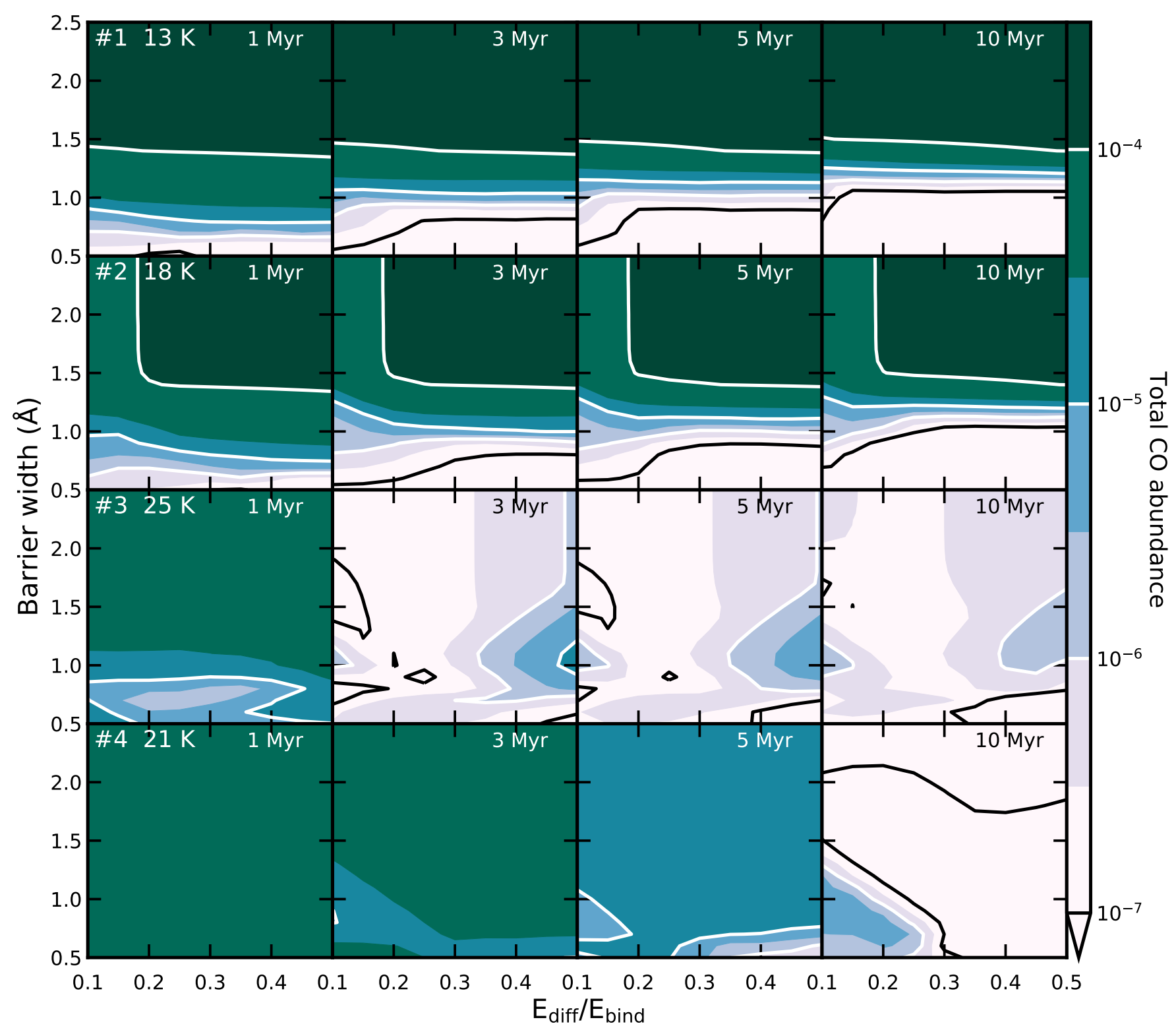

Fig. 6. Time evolution of total $\mathrm{CO}$ abundance (ice and gas) as function of the assumed tunnelling barrier width $\left(a_{\text {tunnel }}\right)$ and diffusion-to-binding energy ratio $\left(f_{\text {diff }}\right)$. All these models have $\zeta_{\mathrm{H}_{2}}=10^{-17} \mathrm{~s}^{-1}$. The first row of models uses $T_{\text {gas }}=13 \mathrm{~K}, n_{\text {gas }}=10^{8} \mathrm{~cm}^{-3}$. The second row of models uses $T_{\text {gas }}=18 \mathrm{~K}$ and $n_{\text {gas }}=3 \times 10^{11} \mathrm{~cm}^{-3}$. The third row of models uses $T_{\text {gas }}=25 \mathrm{~K}$ and $n_{\text {gas }}=5 \times 10^{11} \mathrm{~cm}^{-3}$. The fourth row of models uses $T_{\text {gas }}=21 \mathrm{~K}$ and $n_{\text {gas }}=3 \times 10^{6} \mathrm{~cm}^{-3}$. Significant reduction of the total $\mathrm{CO}$ abundance in less than $3 \mathrm{Myr}$ is only possible if sCO $+\mathrm{sH}$ is efficient, which is at low barrier widths or if sOH preferably reacts with sCO, which is enhanced at low values of $f_{\text {diff }}$. The fiducial values are $a_{\text {tunnel }}=1 \AA$ and $f_{\text {diff }}=0.3$ ).

of the gas phase route starting with dissociative electron transfer of $\mathrm{CO}$ to $\mathrm{He}^{+}: \mathrm{CO}+\mathrm{He}^{+} \longrightarrow \mathrm{C}^{+}+\mathrm{O}+\mathrm{He}$. In the last $3 \mathrm{Myr}$ of chemical evolution, this reaction pathway removes $99.9 \%$ of the $\mathrm{CO}$ in a large part of the parameter space. Only regions that had a significant build up of $\mathrm{CO}_{2}$ at early times, or where $\mathrm{N}_{2}$ can be efficiently reformed, show $\mathrm{CO}$ abundances $>10^{-6}$, because $\mathrm{CO}$ is reformed from $\mathrm{CO}_{2}$ and because $\mathrm{N}_{2}$ competes with $\mathrm{CO}$ for reactions with $\mathrm{He}^{+}$, respectively.

In summary, our results do not strongly depend on the value of $f_{\text {diff }}$; however, the speed of hydrogenation critically depends on the value for $a_{\text {tunnel }}$, especially around $a_{\text {tunnel }}=1 \AA$. Several independent laboratory experiments show that the $\mathrm{CO}$ hydrogenation proceeds fast, even at temperatures as low as 10-12 K (Hiraoka et al. 2002; Watanabe \& Kouchi 2002; Fuchs et al. 2009) so a high barrier is unlikely. Reaction probabilities from the Harmonic Quantum Transition State calculations by
Andersson et al. (2011) are consistent with $a_{\text {tunnel }} \approx 0.9 \AA$ in our calculations. Given the importance of the exact value of the tunnelling barrier on the chemical evolution, more work is needed in understanding the tunnelling of hydrogen during hydrogenation processes.

\section{Discussion}

Our results show that it is possible to chemically process $\mathrm{CO}$ under conditions $\left(T<30 \mathrm{~K}, n=10^{6}-10^{12} \mathrm{~cm}^{-3}\right)$ that are representative of a large mass fraction of a protoplanetary disk on a few Myr timescale. As such, chemical processing of $\mathrm{CO}$, specifically the formation of $\mathrm{CH}_{3} \mathrm{OH}$ and $\mathrm{CO}_{2}$ and on longer timescales hydrocarbons, has a significant effect on the observed $\mathrm{CO}$ abundance. 


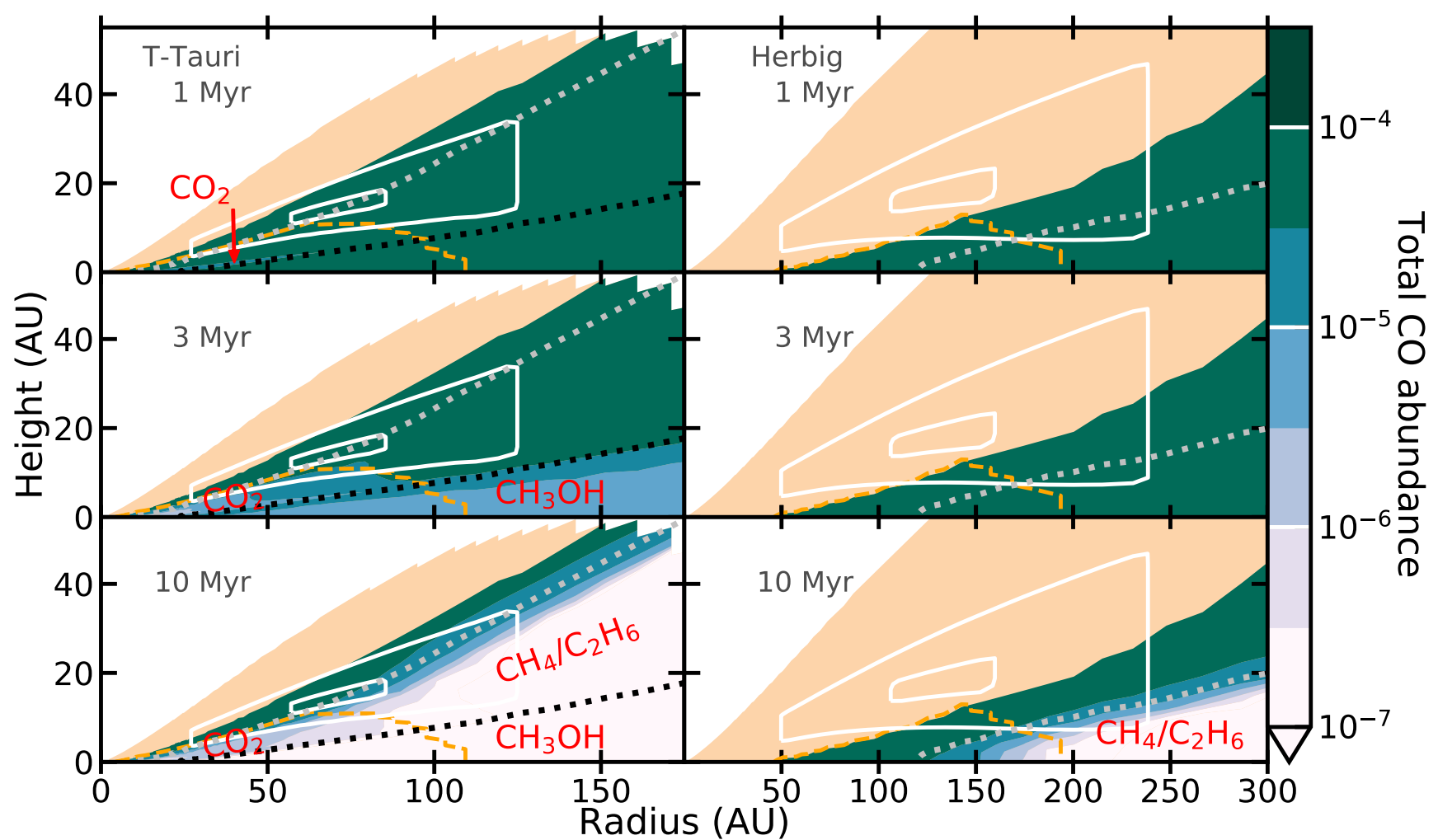

Fig. 7. Total $\mathrm{CO}$ abundance (gas and ice) from the chemical models, at 1, 3 and $10 \mathrm{Myr}$, mapped onto the temperature and density structure of a T Tauri and Herbig disk model (see Fig. A.1 and Appendix A). A cosmic-ray ionisation rate of $10^{-17} \mathrm{~s}^{-1}$ is assumed throughout the disk. The orange area shows the disk region that has a temperature above $40 \mathrm{~K}$ and is thus not included in our chemical models. See Fig. A.1 for the CO abundance in that region using a simple $\mathrm{CO}$ chemistry. The white contours shows the area within which $25 \%$ and $75 \%$ of the $\mathrm{C}^{18} \mathrm{O}$ flux is emitted. The black and grey dotted lines show the 20 and $30 \mathrm{~K}$ contours, respectively. The Herbig disk is always warmer than $20 \mathrm{~K}$. The molecules in red denote the dominant carbon carrier in the regions $\mathrm{CO}$ is depleted. The orange dashed line encompasses the region that is completely shielded from UV radiation (see Sect. 2.1).

Our results agree with Reboussin et al. (2015), Eistrup et al. (2016) and Schwarz et al. (2018) that a significant cosmic-ray ionisation rate, $>5 \times 10^{-18} \mathrm{~s}^{-1}$ in our study, is needed to convert $\mathrm{CO}$.

In contrast with Furuya \& Aikawa (2014) and Yu et al. (2016), we do not find that destruction of CO through reactions with $\mathrm{He}^{+}$is a main pathway for a cosmic-ray ionisation rate of $10^{-17} \mathrm{~s}^{-1}$. This is mostly because this destruction timescale is $>5 \mathrm{Myr}$, for these levels of ionisation. However, Furuya \& Aikawa (2014) assume a higher cosmic-ray ionisation rate $(5 \times$ $10^{-17} \mathrm{~s}^{-1}$ ) whereas Yu et al. (2016) have X-rays that add to the total ionisation rate of the gas. This lowers their $\mathrm{CO}$ destruction timescale significantly (Fig. 3).

\subsection{When, where and how is CO destroyed within $3 \mathrm{Myr}$}

There are two main reaction mechanisms that can destroy $\mathrm{CO}$ in 3 Myr under cold disk midplane conditions, assuming a cosmicray rate of $10^{-17} \mathrm{~s}^{-1}$ : hydrogenation of $\mathrm{CO}$ to $\mathrm{CH}_{3} \mathrm{OH}$ and the formation of $\mathrm{CO}_{2}$. Hydrogenation of $\mathrm{CO}$ forming $\mathrm{CH}_{3} \mathrm{OH}$ reduces $\mathrm{CO}$ by an order of magnitude within $2 \mathrm{Myr}$ in the cold regions of the disk where $\mathrm{CO}$ is completely frozen out (Fig. 5, top two panels; Fig. 7, left column, top two panels). The first step of this pathway $\mathrm{sCO}+\mathrm{sH}$ has a barrier and thus the timescale of this reaction is strongly dependent on the assumed hydrogen tunnelling efficiency $\left(a_{\text {tunnel }}\right)$, with shorter timescales at lower assumed $a_{\text {tunnel }}$ (Fig. 6).

Contrary to Schwarz et al. (2018), we find that $\mathrm{CH}_{3} \mathrm{OH}$ formation is only dominant when $\mathrm{CO}$ is frozen-out, $<20 \mathrm{~K}$. This is probably due to the more complete grain-surface chemistry in our work, allowing other species to react with atomic $\mathrm{H}$ and removing it from the grain-surface before $\mathrm{CO}$ can react with it at higher temperatures. This leads us to conclude that $\mathrm{CO}_{2}$ formation is always the dominant $\mathrm{CO}$ destruction mechanism in the warm molecular layer in the first $5 \mathrm{Myr}$ instead of $\mathrm{CO}$ hydrogenation, showing that a larger grain-surface network needs to be considered.

The second pathway, $\mathrm{CO}+\mathrm{OH} \longrightarrow \mathrm{CO}_{2}$ on the ice, is efficient at slightly higher temperatures, between the $\mathrm{CO}$ iceline temperature and up to $35 \mathrm{~K}$ at the highest densities (Fig. 4). This pathway leads to $\sim 2$ orders decrease in the $\mathrm{CO}$ abundance within $3 \mathrm{Myr}$. The rate of conversion of $\mathrm{CO}$ into $\mathrm{CO}_{2}$ depends strongly on the assumed chemical parameters in the first Myr. By $3 \mathrm{Myr}$ most of these initial differences have been washed out. Only a very slow assumed diffusion rate $\left(f_{\text {bind }}>0.35\right)$ leads to a $\mathrm{CO}$ abundance above $10^{-6}$ after 3 Myr (Fig. 6).

Production of hydrocarbons follows the formation of $\mathrm{CH}_{3} \mathrm{OH}$ in the first Myr. When the $\mathrm{CO}$ abundance has been lowered by 1 to 2 orders of magnitude, production of $\mathrm{CH}_{3} \mathrm{OH}$ stops, while the formation of $\mathrm{CH}_{4}$ continues. At $\sim 3 \mathrm{Myr}, \mathrm{CH}_{4}$ becomes more abundant than $\mathrm{CH}_{3} \mathrm{OH}$. At even longer timescales, $\mathrm{CH}_{4}$ gets turned into $\mathrm{C}_{2} \mathrm{H}_{6}$. Formation of hydrocarbons starting from $\mathrm{CO}+\mathrm{He}^{+}$in the gas phase is only effective at densities $<10^{9} \mathrm{~cm}^{-3}$ between the $\mathrm{CO}$ and $\mathrm{CH}_{4}$ icelines. Even in this region, the timescale is $>3 \mathrm{Myr}$.

The timescales for these processes scale inversely with the cosmic-ray or X-ray ionisation rate. As such, in regions with lower ionisation rates $\mathrm{CO}$ destruction can be slower than described here. The reverse is also true, so if locally produced energetic particles play an significant role, the $\mathrm{CO}$ destruction timescales become shorter. 
In conclusion, for a nominal cosmic-ray ionisation rate of $10^{-17} \mathrm{~s}^{-1}$, CO can be significantly destroyed (orders of magnitude) in the first few Myr of disk evolution under cold conditions. This conclusion is robust against variations in chemical parameters, except if the barrier width for the $\mathrm{CO}+\mathrm{H}$ tunneling becomes too large. Under warm conditions $(T>30 \mathrm{~K})$, the CO abundance is lowered by a factor of a few at most.

\subsection{Implications for observations}

Mapping the results from the chemical models summarized above onto the physical structure seen in Fig. 1 results in Fig. 7. It is clear that $\mathrm{CO}$ can be destroyed over a region of the disk, that contains a significant amount of mass. For the T Tauri disk a portion of this $\mathrm{CO}$ destruction will be in a region of the disk where $\mathrm{CO}$ is not traceable by observations, as the $\mathrm{CO}$ is frozen out. However, there is also a significant region up to $30 \mathrm{~K}$ within and above the $\mathrm{CO}$ snow surface where $\mathrm{CO}$ is depleted and where the emission from the less abundant ${ }^{18} \mathrm{O}$ and ${ }^{17} \mathrm{O}$ isotopologues originates (Miotello et al. 2014). Thus, the processes studied here can explain, at least in part, the observations of low CO abundances within the iceline in TW Hya (Zhang et al. 2017).

If $\mathrm{CO}$ destruction would also be effective in disk regions that are irradiated by moderate amounts $\left(<1 G_{0}\right)$ of (interstellar) UV radiation, then a region responsible for $\sim 20 \%$ of the emission can be depleted in CO by $\sim 1$ order of magnitude by $3 \mathrm{Myr}$, as seen in Fig. 7. The other $\sim 80 \%$ is also likely affected due to mixing of $\mathrm{CO}$ poor gas from below the iceline into the emitting region (see Sect. 4.4). This would result in a drop of the $\mathrm{C}^{18} \mathrm{O}$ isotopologue flux between $20 \%$ and an order of magnitude depending on the vertical mixing efficiency. Rarer isotopologues, whose flux comes from lower, colder layers, would be more severely affected. The T Tauri model shown in Figs. 1 and 7 assume a total source luminosity of around $0.3 L_{\odot}$ representative of the bulk of $\mathrm{T}$ Tauri stars in low mass star-forming regions (Alcalá et al. 2017). For disks around stars with a lower luminosity or with higher ages, the effect of $\mathrm{CO}$ destruction would be even larger.

The warm upper layers exposed to modest UV have also been modelled by Schwarz et al. (2018). They find that the CO abundance is generally high in these intermediate layers, with significant CO destruction only taking place for high X-ray or cosmic ray ionisation rates. This is consistent with our Figs. 7 and A.1.

For Herbig sources, only a small region of the disk falls within the range of parameters studied here and an even smaller region is at temperatures under $30 \mathrm{~K}$, while also being shielded (Fig. 1). Because Herbig disks host more luminous stars than their $\mathrm{T}$ Tauri counterparts, they also tend to be warmer. This means that a smaller mass fraction of the disk has temperatures between 10 and $30 \mathrm{~K}$, where $\mathrm{CO}$ can be efficiently destroyed. As such our chemical models predict that Herbig disk have a $\mathrm{CO}$ abundance that is close to canonical, consistent with observations by Kama et al. (2016).

Young disks around T Tauri stars are expected to be warmer due to the accretional heating (Harsono et al. 2015). They are also younger than the time needed to significantly convert $\mathrm{CO}$ to other species with grain surface reactions. As such young disks should close to canonical $\mathrm{CO}$ abundances, as seems to be observed for at least some disks (van 't Hoff et al. 2018).

\subsection{Observing chemical destruction of $\mathrm{CO}$}

In our models, $\mathrm{CO}$ is mostly processed into species that are frozen-out in most of the disk, complicating detection of these products with sub-mm lines. Several of the prominent carbon reservoirs, like $\mathrm{CO}_{2}, \mathrm{CH}_{4}$ and $\mathrm{C}_{2} \mathrm{H}_{6}$, are symmetric molecules without a dipole moment, so they do not have detectable sub-mm lines. Even $\mathrm{CH}_{3} \mathrm{OH}$, which does have strong microwave transitions, is difficult to detect since the processes that get methanol ice off the grains mostly destroy the molecule (e.g. Bertin et al. 2016; Walsh et al. 2016). Thus, observing the molecules in the solid state would be the best proof of this chemical processing. Another way to indirectly observe the effects chemical processing of $\mathrm{CO}$ would be to compare to cometary abundances. This will be discussed in Sect. 4.4.

Ice does not emit strong mid-infrared bands. However, for some highly inclined disks, the outer disk ice content can be probed with infrared absorption against the strong mid-infrared continuum of the inner disk with the line of sight passing through the intermediate height disk layers (Pontoppidan et al. 2005). The processes discussed here will leave a chemical imprint on the ice absorption spectra. The destruction pathways of $\mathrm{CO}$ preferentially create $\mathrm{CO}_{2}, \mathrm{CH}_{3} \mathrm{OH}, \mathrm{CH}_{4}$ and $\mathrm{H}_{2} \mathrm{O} . \mathrm{CO}_{2}$ reaches a peak abundance of $\sim 7 \times 10^{-5}$, seven times higher than the initial conditions, which would be detectable. A large $\mathrm{CO}_{2}$ ice reservoir would therefore immediately point at a conversion of $\mathrm{CO}$ to $\mathrm{CO}_{2}$, either in the disk or en route to the disk (Drozdovskaya et al. 2016). The formation of $\mathrm{CH}_{4}$ and $\mathrm{CH}_{3} \mathrm{OH}$ happens mostly in the coldest regions of the disk $(<20 \mathrm{~K})$ as such observations of large fractions $\left(>20 \%\right.$ with respect to $\left.\mathrm{H}_{2} \mathrm{O}\right)$ of $\mathrm{CH}_{4}$ and $\mathrm{CH}_{3} \mathrm{OH}$ in the ice would point at a disk chemistry origin of $\mathrm{CO}$ destruction. If large amounts of $\mathrm{CH}_{4}$ or $\mathrm{CH}_{3} \mathrm{OH}$ are found in infrared absorption spectra, this would also imply that vertical mixing plays a role in the chemical processing, as this is needed to move the $\mathrm{CH}_{4}$ and/or $\mathrm{CH}_{3} \mathrm{OH}$ ice hosting grains away from the cold midplane up to the layers that can be probed with infrared observations. For $\mathrm{H}_{2} \mathrm{O}$ the increase is generally smaller than a factor of two, so that $\mathrm{H}_{2} \mathrm{O}$ ice is not a good tracer of $\mathrm{CO}$ destruction.

If the gas and dust in the midplane is not static, but moves towards the star due to the accretion flow or radial drift of dust grains, then it is expected that the products of $\mathrm{CO}$ destruction are thermally desorbed in the inner disk (see Booth et al. 2017; Bosman et al. 2018). This would cause an observable increase in abundance. Current infrared observations of gaseous $\mathrm{CO}_{2}$ emission do not show a signal consistent with an $\mathrm{CO}_{2}$ enriched inner disk, but the optical thickness of the $15 \mu \mathrm{m} \mathrm{CO}_{2}$ lines in the surface layers might hinder the observation of $\mathrm{CO}_{2}$ near the disk midplane (Salyk et al. 2011; Pontoppidan \& Blevins 2014; Walsh et al. 2015; Bosman et al. 2017, 2018). Gibb \& Horne (2013) have observed gaseous $\mathrm{CH}_{4}$ in absorption in the near-infrared in GV Tau N. Their probed $\mathrm{CH}_{4}$ is rotationally hot $(750 \mathrm{~K})$ and is thus likely situated within the inner $1 \mathrm{AU}$ of the disk. They rule out a large column of $\mathrm{CH}_{4}$ at lower temperatures (100 K). However, this disk is still embedded, so it is possibly too young to have converted $\mathrm{CO}$ into $\mathrm{CH}_{4}$ (Carney et al. 2016). Gaseous $\mathrm{CH}_{4}$ and $\mathrm{CH}_{3} \mathrm{OH}$ have not been detected with Spitzer-IRS in disks, but should be detectable with JWST-MIRI. If lines from these molecules are brighter than expected for inner disk chemistry, it could point at a scenario in which $\mathrm{CO}$ is converted to $\mathrm{CH}_{4}$ and $\mathrm{CH}_{3} \mathrm{OH}$ in the cold $(<20 \mathrm{~K})$ outer-midplane regions of the disk, and that the reaction products are brought into the inner disk via accretion or radial drift.

\subsection{Interactions with disk dynamics}

The chemical timescales needed for an order of magnitude decrease of the $\mathrm{CO}$ dependence are close to 2 Myr. This timescale is significantly longer than the turbulent mixing timescales, even in the outer disk. As such it is not inconceivable that either vertical mixing or gas accretion influences the 
abundance of $\mathrm{CO}$. For both the $\mathrm{sCO}+\mathrm{sH}$ and the $\mathrm{sCO}+\mathrm{sOH}$ route, the rate scales with the abundance of $\mathrm{CO}$ in the ice: the higher the abundance, the faster the rate, although the dependence can be sub-linear. This means that, if vertical mixing replenishes the disk midplane $\mathrm{CO}$ reservoir, the destruction of CO can happen at a higher rate for a longer time. This converts $\mathrm{CO}$ to other species not only in the regions where the processes described in this paper are effective but also in the regions around it. For mixing to have a significant effect at $100 \mathrm{AU}$, a turbulent $\alpha$ of $10^{-4}$ or higher is needed (see, e.g., Ciesla 2010; Semenov \& Wiebe 2011; Bosman et al. 2018).

The freeze-out of $\mathrm{CO}$ on grains that are large enough to have settled below the $\mathrm{CO}$ snow surface can also lower the abundance of $\mathrm{CO}$ in the disk atmosphere. This process is thought to happen for $\mathrm{H}_{2} \mathrm{O}$ to explain the low observed abundances of $\mathrm{H}_{2} \mathrm{O}$ in the outer disk (Hogerheijde et al. 2011; Krijt et al. 2016; Du et al. 2017). Using a toy model Kama et al. (2016) showed that the $\mathrm{CO}$ abundance in the disk atmosphere can be lowered by 1-2 orders of magnitude in the disk lifetime. This process can work in concert with the chemical destruction of $\mathrm{CO}$ to lower the $\mathrm{CO}$ abundance in the outer disk. If large grains are locking up a significant fraction of the $\mathrm{CO}$ near the midplane outside of the $\mathrm{CO}$ iceline, then this $\mathrm{CO}$ would come off the grains near the $\mathrm{CO}$ iceline, leading to an strong increase in the $\mathrm{CO}$ abundance. Zhang et al. (2017) show however that for TW Hya, this is not the case: $\mathrm{CO}$ also has a low abundance of $\sim 3 \times 10^{-6}$ within the $\mathrm{CO}$ iceline. Further observations will have to show if this is the case for all disks with a low CO abundance, or if TW Hya is the exception and this low $\mathrm{CO}$ abundance is caused by its exceptional age of $\sim 8 \mathrm{Myr}$ (Donaldson et al. 2016).

If the destruction of $\mathrm{CO}$ within the $\mathrm{CO}$ snowline is a general feature of protoplanetary disks, then a mechanism to stop any $\mathrm{CO}$ locked up in grains from being released in the gas phase will need to be considered. This is hard to do without actually halting radial drift completely, for the same reasons that it is hard to stop $\mathrm{CO}_{2}$ from desorbing off the grain at its respective snowline (Bosman et al. 2018).

The fact that comets in the solar system do contain significant amounts of $\mathrm{CO}$ (up to $30 \%$ with respect to $\mathrm{H}_{2} \mathrm{O}$; Mumma \& Charnley 2011; Le Roy et al. 2015) suggests that these comets were formed in a cold $\mathrm{CO}$ rich environment, possibly before the bulk of the $\mathrm{CO}$ was converted into other molecules. In other disks, such CO-rich grains must then have been trapped outside the $\mathrm{CO}$ iceline to prevent significant $\mathrm{CO}$ sublimating in the inner regions.

\section{Conclusions}

We performed a kinetic chemical modelling study of the destruction of $\mathrm{CO}$ under UV shielded, cold $(<40 \mathrm{~K})$ and dense $\left(10^{6}-10^{12} \mathrm{~cm}^{-3}\right)$ conditions. Both grain-surface and gas phase routes to destroy $\mathrm{CO}$ are considered and their efficiencies and timescales evaluated using a gas-grain chemical network. Furthermore, we studied the effects of the assumed ice diffusion speed (through the diffusion-to-binding energy ratio, $f_{\text {diff }}$ ) and the assumed $\mathrm{H}$ and $\mathrm{H}_{2}$ tunnelling efficiency (through the tunnelling barrier width, $\left.a_{\text {tunnel }}\right)$ on the evolution of the $\mathrm{CO}$ abundance both in the gas phase and on the grain-surface. Our findings can be summarised as follows:

- CO destruction is linearly dependent on the assumed $\mathrm{H}_{2}$ ionisation rate by energetic particles (cosmic rays, $\mathrm{X}$-rays) over a large region of the considered physical parameter space. Only high enough cosmic-ray ionisation rates, $>5 \times 10^{-18} \mathrm{~s}^{-1}$ can destroy CO on a $<3$ Myr timescale (Sect. 3.1.1, Fig. 3).
- The chemical processing of CO is most efficient at low temperatures. A relation between disk temperature and measured $\mathrm{CO}$ abundance is expected. The coldest disks would have the lowest $\mathrm{CO}$ abundances; in contrast, flaring disks around luminous Herbig stars should have close to canonical $\mathrm{CO}$ abundances.

- At low temperatures, hydrogenation of $\mathrm{CO}$ is efficient when $\mathrm{CO}$ is fully frozen-out, leading to a reduction of the total $\mathrm{CO}$ abundance by $\sim 2$ orders assuming $\zeta_{\mathrm{H}_{2}}=10^{-17} \mathrm{~s}^{-1}$. This route is only weakly dependent on the temperature and density, as long as CO is fully frozen-out (Sect. 3.1, Fig. 4).

- At temperatures of 20-30 K, just above the desorption temperature of $\mathrm{CO}$, formation of $\mathrm{CO}_{2}$ from the reaction of $\mathrm{CO}$ with $\mathrm{OH}$ on the ice is efficient. The $\mathrm{CO}$ abundance can be reduced by two orders of magnitude in 2-3 Myr for $\zeta_{\mathrm{H}_{2}}=10^{-17} \mathrm{~s}^{-1}$. The formation of $\mathrm{CO}_{2}$ is more efficient at higher densities (Sect. 3.1, Fig. 4).

- Gas phase destruction of $\mathrm{CO}$ by $\mathrm{He}^{+}$, eventually leading to the formation $\mathrm{CH}_{4}$ and $\mathrm{H}_{2} \mathrm{O}$, only operates on timescales $>5 \mathrm{Myr}$ for $\zeta_{\mathrm{H}_{2}}=10^{-17} \mathrm{~s}^{-1}$. Furthermore, this pathway is only effective at low densities $\left(<10^{9} \mathrm{~cm}^{-3}\right)$ and in a small range of temperatures $(15-25 \mathrm{~K})$. As such, this pathway is not important in the context of protoplanetary disk midplanes (Sect. 3.1, Fig. 4).

- The assumed tunnelling barrier width $\left(a_{\text {tunnel }}\right)$ strongly influences the speed of $\mathrm{CO}$ hydrogenation with efficient tunnelling leading to fast hydrogenation of $\mathrm{CO}$. The $\mathrm{CO}$ destruction due to $\mathrm{CO}_{2}$ formation is only weakly dependent on the assumed chemical parameters. Only when $f_{\text {diff }}$ is increased above 0.35 can this reaction be slowed down (Sect. 3.2, Fig. 6).

- $\mathrm{CO}_{2}, \mathrm{CH}_{3} \mathrm{OH}$ and, on a longer timescale, $\mathrm{CH}_{4}$ are all abundantly formed in the regions where $\mathrm{CO}$ is destroyed. Observations of anomalously high abundances of $\mathrm{CH}_{4}, \mathrm{CH}_{3} \mathrm{OH}$ or $\mathrm{CO}_{2}$ either in infrared absorption spectroscopy towards edge-on systems, or in infrared emission from the inner disk, can help in distinguishing the chemical pathway responsible for $\mathrm{CO}$ destruction.

- Vertical mixing can bring gas from the warmer, $\mathrm{CO}$ richer layers to the lower colder layers, where $\mathrm{CO}$ can be converted into $\mathrm{CH}_{3} \mathrm{OH}$ or $\mathrm{CO}_{2}$. This would allow for the chemical processes to also lower the $\mathrm{CO}$ abundance in the higher, warmer layers of the disk.

In conclusion, chemical reprocessing of $\mathrm{CO}$ can have a significant impact on the measured disk masses if sufficient ionising radiation is present in the cold $(<30 \mathrm{~K})$ regions of the disk. Further modelling of individual disks will have to show to which degree chemical processes are important and where other physical processes will need to be invoked.

Acknowledgements. Astrochemistry in Leiden is supported by the European Union A-ERC grant 291141 CHEMPLAN, by the Netherlands Research School for Astronomy (NOVA), by a Royal Netherlands Academy of Arts and Sciences (KNAW) professor prize. C.W. acknowledges the University of Leeds for financial support.

\section{References}

Acharyya, K., Fuchs, G. W., Fraser, H. J., van Dishoeck, E. F., \& Linnartz, H. 2007, A\&A, 466, 1005

Aikawa, Y., Umebayashi, T., Nakano, T., \& Miyama, S. M. 1999, ApJ, 519, 705

Alcalá, J. M., Manara, C. F., Natta, A., et al. 2017, A\&A, 600, A20

Andersson, S., Goumans, T. P. M., \& Arnaldsson, A. 2011, Chem. Phys. Lett., 513,31

Ansdell, M., Williams, J. P., van der Marel, N., et al. 2016, ApJ, 828, 46

Arasa, C., van Hemert, M. C., van Dishoeck, E. F., \& Kroes, G. J. 2013, J. Phys. Chem. A, 117, 7064 
Baruteau, C., Crida, A., Paardekooper, S.-J., et al. 2014, Protostars and Planets VI, 667

Bergin, E. A., Cleeves, L. I., Gorti, U., et al. 2013, Nature, 493, 644

Bergin, E. A., \& Williams, J. P. 2017, in Astrophysics and Space Science Library, eds. M. Pessah \& O. Gressel, 445, 1

Bertin, M., Romanzin, C., Doronin, M., et al. 2016, ApJ, 817, L12

Birnstiel, T., Dullemond, C. P., \& Brauer, F. 2010, A\&A, 513, A79

Bolatto, A. D., Wolfire, M., \& Leroy, A. K. 2013, ARA\&A, 51, 207

Boogert, A. C. A., Gerakines, P. A., \& Whittet, D. C. B. 2015, ARA\&A, 53, 541

Booth, R. A., Clarke, C. J., Madhusudhan, N., \& Ilee, J. D. 2017, MNRAS, 469 3994

Bosman, A. D., Bruderer, S., \& van Dishoeck E. F. 2017, A\&A, 601, A36

Bosman, A. D., Tielens, A. G. G. M., \& van Dishoeck E. F. 2018, A\&A, 611, A80

Brauer, F., Dullemond, C. P., \& Henning, T. 2008, A\&A, 480, 859

Brown, W. A., \& Bolina, A. S. 2007, MNRAS, 374, 1006

Bruderer, S. 2013, A\&A, 559, A46

Bruderer, S., Doty, S. D., \& Benz, A. O. 2009, ApJS, 183, 179

Bruderer, S., van Dishoeck, E. F., Doty, S. D., \& Herczeg, G. J. 2012, A\&A, 541 A91

Carney, M. T., Yıldız, U. A., Mottram, J. C., et al. 2016, A\&A, 586, A44

Cazaux, S., \& Tielens, A. G. G. M. 2004, ApJ, 604, 222

Chen, Y.-J., Chuang, K.-J., Muñoz Caro, G. M., et al. 2014, ApJ, 781, 15

Ciesla, F. J. 2010, ApJ, 723, 514

Cleeves, L. I., Bergin, E. A., Qi, C., Adams, F. C., \& Öberg, K. I. 2015, ApJ, 799, 204

Collings, M. P., Dever, J. W., Fraser, H. J., \& McCoustra, M. R. S. 2003, Ap\&SS, 285,633

Cooke, I. R., Öberg, K. I., Fayolle, E. C., Peeler, Z., \& Bergner, J. B. 2018, ApJ, 852,75

Cuppen, H. M., \& Herbst, E. 2007, ApJ, 668, 294

Cuppen, H. M., van Dishoeck, E. F., Herbst, E., \& Tielens, A. G. G. M. 2009, A\&A, 508, 275

Cuppen, H. M., Ioppolo, S., Romanzin, C., \& Linnartz, H. 2010, Phys. Chem. Chem. Phys., 12, 12077

Cuppen, H. M., Walsh, C., Lamberts, T., et al. 2017, Space Sci. Rev., 212, 1

Dalgarno, A. 2006, Proc. Natl. Acad. Sci., 103, 12269

Donaldson, J. K., Weinberger, A. J., Gagné, J., et al. 2016, ApJ, 833, 95

Draine, B. T. 1978, ApJS, 36, 595

Draine, B. T., \& Lee, H. M. 1984, ApJ, 285, 89

Drozdovskaya, M. N., Walsh, C., van Dishoeck, E. F., et al. 2016, MNRAS, 462 977

Du, F., Bergin, E. A., \& Hogerheijde, M. R. 2015, ApJ, 807, L32

Du, F., Bergin, E. A., Hogerheijde, M., et al. 2017, ApJ, 842, 98

Dutrey, A., Guilloteau, S., \& Guelin, M. 1997, A\&A, 317, L55

Eistrup, C., Walsh, C., \& van Dishoeck E. F. 2016, A\&A, 595, A83

Eistrup, C., Walsh, C., \& van Dishoeck E. F. 2018, A\&A, 613, A14

Favre, C., Cleeves, L. I., Bergin, E. A., Qi, C., \& Blake, G. A. 2013, ApJ, 776, L38

Fayolle, E. C., Öberg, K. I., Cuppen, H. M., Visser, R., \& Linnartz, H. 2011, A\&A, 529, A74

Fuchs, G. W., Cuppen, H. M., Ioppolo, S., et al. 2009, A\&A, 505, 629

Furuya, K., \& Aikawa, Y. 2014, ApJ, 790, 97

Furuya, K., Aikawa, Y., Hincelin, U., et al. 2015, A\&A, 584, A124

Furuya, K., van Dishoeck, E. F., \& Aikawa, Y. 2016, A\&A, 586, A127

Garrod, R. T., \& Herbst, E. 2006, A\&A, 457, 927

Garrod, R. T., \& Pauly, T. 2011, ApJ, 735, 15

Garrod, R. T., Widicus Weaver, S. L., \& Herbst, E. 2008, ApJ, 682, 283

Gibb, E. L., \& Horne, D. 2013, ApJ, 776, L28

Harsono, D., Bruderer, S., \& van Dishoeck E. F. 2015, A\&A, 582, A41

Hasegawa, T. I., Herbst, E., \& Leung, C. M. 1992, ApJS, 82, 167

He, J., \& Vidali, G. 2014, ApJ, 788, 50

Hincelin, U., Chang, Q., \& Herbst, E. 2015, A\&A, 574, A24

Hindmarsh, A. C., Brown, P. N., Grant, K. E., et al. 2005, ACM Trans. Math. Softw., 31, 363

Hiraoka, K., Sato, T., Sato, S., et al. 2002, ApJ, 577, 265

Hogerheijde, M. R., Bergin, E. A., Brinch, C., et al. 2011, Science, 334, 338

Ioppolo, S., Fedoseev, G., Lamberts, T., Romanzin, C., \& Linnartz, H. 2013, Rev. Sci. Instrum., 84, 073112

Johansen, A., Blum, J., Tanaka, H., et al. 2014, in Protostars and Planets VI, eds H. Beuther, R. Klessen, C. Dullemond, \& T. Henning (Tucson: University of Arizona Press), 547

Kama, M., Bruderer, S., van Dishoeck, E. F., et al. 2016, A\&A, 592, A83

Kley, W., \& Nelson, R. P. 2012, ARA\&A, 50, 211

Krijt, S., Ciesla, F. J., \& Bergin, E. A. 2016, ApJ, 833, 285

Lamberts, T., Cuppen, H. M., Ioppolo, S., \& Linnartz, H. 2013, Phys. Chem. Chem. Phys., 15, 8287
Lauck, T., Karssemeijer, L., Shulenberger, K., et al. 2015, ApJ, 801, 118 Le Roy, L., Altwegg, K., Balsiger, H., et al. 2015, A\&A, 583, A1

Lee, H.-H., Herbst, E., Pineau des Forets, G., Roueff, E., \& Le Bourlot, J. 1996, A\&A, 311, 690

Long, F., Herczeg, G. J., Pascucci, I., et al. 2017, ApJ, 844, 99

Manara, C. F., Rosotti, G., Testi, L., et al. 2016, A\&A, 591, L3

McClure, M. K., Bergin, E. A., Cleeves, L. I., et al. 2016, ApJ, 831, 167

McElroy, D., Walsh, C., Markwick, A. J., et al. 2013, A\&A, 550, A36

Miotello, A., Bruderer, S., \& van Dishoeck E. F. 2014, A\&A, 572, A96

Miotello, A., van Dishoeck, E. F., Kama, M., \& Bruderer, S. 2016, A\&A, 594, A85

Miotello, A., van Dishoeck, E. F., Williams, J. P., et al. 2017, A\&A, 599, A113

Molyarova, T., Akimkin, V., Semenov, D., et al. 2017, ApJ, 849, 130

Muñoz Caro, G. M., Jiménez-Escobar, A., Martín-Gago, J. Á., et al. 2010, A\&A, 522, A 108

Muñoz Caro, G. M., Chen, Y.-J., Aparicio, S., et al. 2016, A\&A, 589, A19

Mumma, M. J., \& Charnley, S. B. 2011, ARA\&A, 49, 471

Noble, J. A., Congiu, E., Dulieu, F., \& Fraser, H. J. 2012, MNRAS, 421, 768

Oba, Y., Watanabe, N., Kouchi, A., Hama, T., \& Pirronello, V. 2010, ApJ, 712, L174

Öberg, K. I., Fuchs, G. W., Awad, Z., et al. 2007, ApJ, 662, L23

Öberg, K. I., Garrod, R. T., van Dishoeck, E. F., \& Linnartz, H. 2009a, A\&A 504,891

Öberg, K. I., Linnartz, H., Visser, R., \& van Dishoeck E. F. 2009b, ApJ, 693 , 1209

Öberg, K. I., van Dishoeck, E. F., \& Linnartz, H. 2009c, A\&A, 496, 281

Paardekooper, D. M., Fedoseev, G., Riedo, A., \& Linnartz, H. 2016, A\&A, 596, A72

Pascucci, I., Testi, L., Herczeg, G. J., et al. 2016, ApJ, 831, 125

Penteado, E. M., Walsh, C., \& Cuppen, H. M. 2017, ApJ, 844, 71

Pontoppidan, K. M., \& Blevins, S. M. 2014, Faraday Discuss., 169, 49

Pontoppidan, K. M., Dullemond, C. P., van Dishoeck, E. F., et al. 2005, ApJ, 622, 463

Pontoppidan, K. M., Boogert, A. C. A., Fraser, H. J., et al. 2008, ApJ, 678, 1005

Reboussin, L., Wakelam, V., Guilloteau, S., Hersant, F., \& Dutrey, A. 2015, A\&A, 579, A82

Salyk, C., Pontoppidan, K. M., Blake, G. A., Najita, J. R., \& Carr, J. S. 2011, ApJ, 731, 130

Schwarz, K. R., Bergin, E. A., Cleeves, L. I., et al. 2016, ApJ, 823, 91

Schwarz, K. R., Bergin, E. A., Cleeves, L. I., et al. 2018, ApJ, 856, 85

Semenov, D., \& Wiebe, D. 2011, ApJS, 196, 25

Senevirathne, B., Andersson, S., Dulieu, F., \& Nyman, G. 2017, Mol. Astrophys., 6,59

Shen, C. J., Greenberg, J. M., Schutte, W. A., \& van Dishoeck E. F. 2004, A\&A, 415,203

Taquet, V., Ceccarelli, C., \& Kahane, C. 2012, A\&A, 538, A42

Tielens, A. G. G. M., \& Hagen, W. 1982, A\&A, 114, 245

Tielens, A. G. G. M., Tokunaga, A. T., Geballe, T. R., \& Baas, F. 1991, ApJ, 381 , 181

Trapman, L., Miotello, A., Kama, M., van Dishoeck, E. F., \& Bruderer, S. 2017, A\&A, 605, A69

van Dishoeck, E. F., \& Black, J. H. 1987, in Physical Processes in Interstellar Clouds, ed. G. E. Morfill \& M. Scholer, in NATO ASIC Proc., 210, 241

van Dishoeck, E. F., \& Black, J. H. 1988, ApJ, 334, 771

van 't Hoff, M. L. R., Tobin, J. J., Harsono, D., \& van Dishoeck E. F. 2018, A\&A, 615, A83

van Zadelhoff, G.-J., van Dishoeck, E. F., Thi, W.-F., \& Blake, G. A. 2001, A\&A, 377,566

van Zadelhoff, G.-J., Aikawa, Y., Hogerheijde, M. R., \& van Dishoeck E. F. 2003 , A\&A, 397, 789

Viala, Y. P., Letzelter, C., Eidelsberg, M., \& Rostas, F. 1988, A\&A, 193, 265

Visser, R., van Dishoeck, E. F., \& Black, J. H. 2009, A\&A, 503, 323

Wakelam, V., Ruaud, M., Hersant, F., et al. 2016, A\&A, 594, A35

Walsh, C., Millar, T. J., \& Nomura, H. 2010, ApJ, 722, 1607

Walsh, C., Nomura, H., Millar, T. J., \& Aikawa, Y. 2012, ApJ, 747, 114

Walsh, C., Nomura, H., \& van Dishoeck E. 2015, A\&A, 582, A88

Walsh, C., Loomis, R. A., Öberg, K. I., et al. 2016, ApJ, 823, L10

Watanabe, N., \& Kouchi, A. 2002, ApJ, 571, L173

Watanabe, N., Mouri, O., Nagaoka, A., et al. 2007, ApJ, 668, 1001

Weidenschilling, S. J. 1977, MNRAS, 180, 57

Weingartner, J. C., \& Draine, B. T. 2001, ApJ, 548, 296

Williams, J. P., \& Best, W. M. J. 2014, ApJ, 788, 59

Woon, D. E. 2002, ApJ, 569, 541

Yu, M., Willacy, K., Dodson-Robinson, S. E., Turner, N. J., \& Evans, II, N. J. 2016, ApJ, 822, 53

Zhang, K., Bergin, E. A., Blake, G. A., Cleeves, L. I., \& Schwarz, K. R. 2017, Nat. Astron., 1, 0130 


\section{Appendix A: Dali protoplanetary disk models}

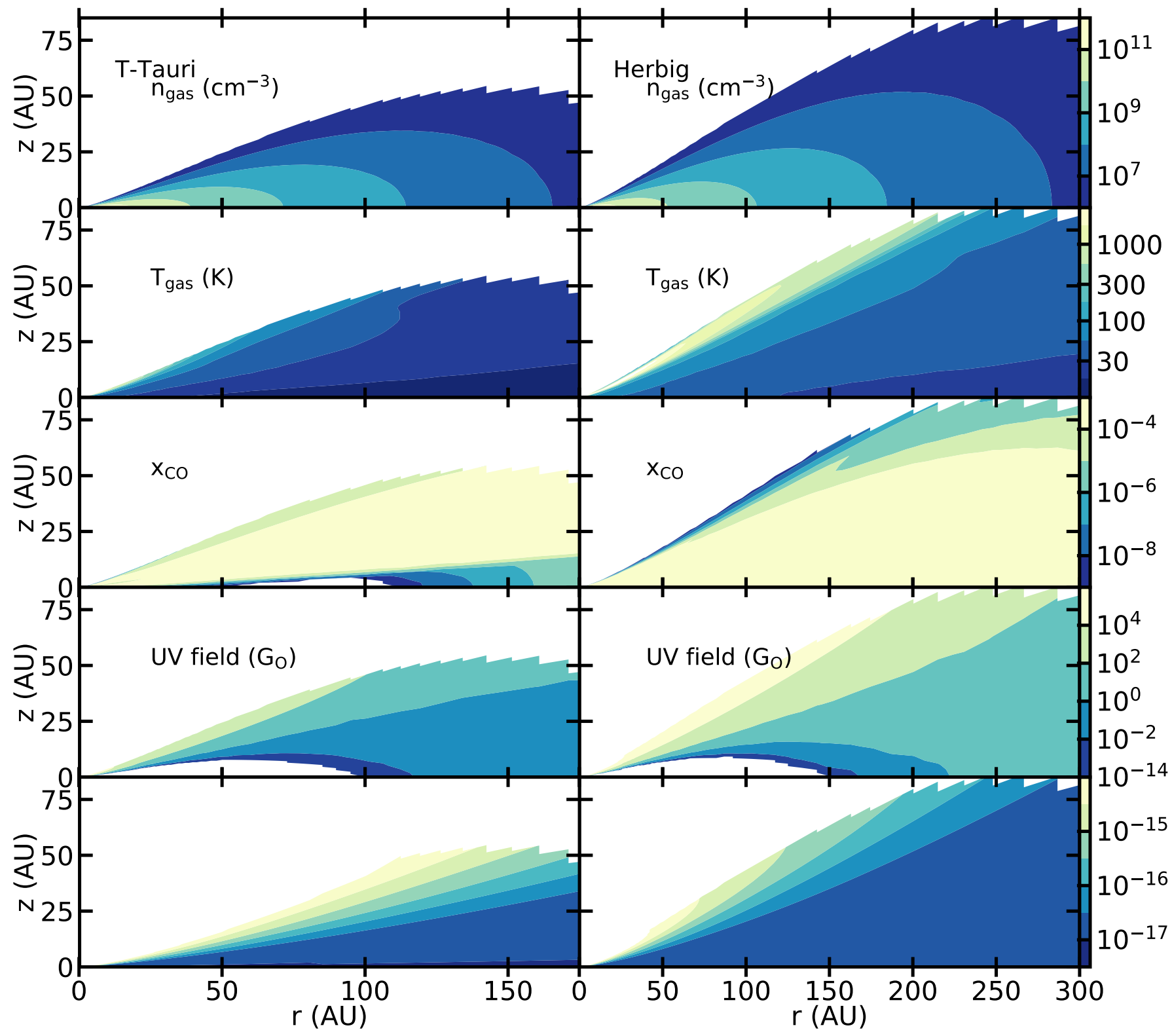

Fig. A.1. Gas number density, gas temperature, gas phase CO fractional abundance and UV radiation field for the T Tauri and Herbig models. A full gas-grain model was not considered for the $\mathrm{CO}$ abundance. Freeze-out, desorption, gas phase reactions and photodissociation are included in the chemical computation. $\mathrm{CO}$ abundance after $1 \mathrm{Myr}$ of chemical evolution is shown.

For Fig. 1 two DALI models (Bruderer et al. 2012; Bruderer 2013) have been run to generate maps of the temperature and radiation field for the disks given the gas and dust density structures and stellar spectra as inputs. The input parameters can be found in Table A.1. In the following, the modelling procedure is shortly reiterated.

Radially the gas and dust are distributed using a tapered power law,

$\left.\Sigma_{\text {gas }}=\Sigma_{c}\left(\frac{R}{R_{c}}\right)\right)^{-\gamma} \exp \left[-\left(\frac{R}{R_{c}}\right)^{2-\gamma}\right]$

$\left.\Sigma_{\text {dust }}=\frac{\Sigma_{c}}{g / d}\left(\frac{R}{R_{c}}\right)\right)^{-\gamma} \exp \left[-\left(\frac{R}{R_{c}}\right)^{2-\gamma}\right]$.

Vertically the gas and dust are distributed according to a Gaussian, the dust is divided into a "large" grain and a "small" grain population (see Table A.1), the large grain population contains a fraction $f_{\text {large }}$ of the mass.

$\rho_{\text {gas }}(R, \Theta)=\frac{\Sigma_{\text {gas }}(R)}{\sqrt{2 \pi} R h_{\text {gas }}(R)} \exp \left[-\frac{1}{2}\left(\frac{\pi / 2-\Theta}{h(R)}\right)^{2}\right]$,

$\rho_{\text {dust, small }}(R, \Theta)=\frac{\left(1-f_{\text {large }}\right) \Sigma_{\text {dust }}(R)}{\sqrt{2 \pi} R h_{\text {gas }}(R)} \exp \left[-\frac{1}{2}\left(\frac{\pi / 2-\Theta}{h_{\text {gas }}(R)}\right)^{2}\right]$,

$\rho_{\text {dust, large }}(R, \Theta)=\frac{f_{\text {large }} \Sigma_{\text {gas }}(R)}{\sqrt{2 \pi} R h_{\text {large }}(R)} \exp \left[-\frac{1}{2}\left(\frac{\pi / 2-\Theta}{h_{\text {large }}(R)}\right)^{2}\right]$,

where $h_{\text {gas }}(R)=h_{c}\left(R / R_{c}\right)^{\psi}$ and $h_{\text {large }}(R)=\chi h_{\text {gas }}(R)$. The smaller scale height for the larger grains mimics a degree of settling. 
Table A.1. Adopted model parameters for T Tauri and Herbig disks.

\begin{tabular}{llcc}
\hline \hline Parameter & & T Tauri & Herbig \\
\hline Star & & & \\
Mass & $M_{\star}\left[M_{\odot}\right]$ & 0.2 & 2.5 \\
Luminosity & $L_{\star}\left[L_{\odot}\right]$ & 0.3 & 20 \\
Effective temperature & $T_{\text {eff }}[\mathrm{K}]$ & 3500 & 10000 \\
Accretion luminosity & $L_{\text {accr }}\left[L_{\odot}\right]$ & 0.04 & 0 \\
Accretion temperature & $T_{\text {accr }}[\mathrm{K}]$ & 10000 & - \\
Disk & & & \\
Disk Mass $(g / d=100)$ & $M_{\text {disk }}\left[M_{\odot}\right]$ & 0.01 & 0.024 \\
Surface density index & $\gamma$ & 1.0 & 1.0 \\
Characteristic radius & $R_{c}[\mathrm{AU}]$ & 35 & 75 \\
Inner radius & $R_{\text {in }}[\mathrm{AU}]$ & 0.07 & 0.221 \\
Scale height index & $\psi$ & 0.3 & 0.3 \\
Scale height angle & $h_{c}[\mathrm{rad}]$ & 0.1 & 0.1 \\
Large grain height scaling & $\chi$ & 0.2 & 0.2 \\
Dust properties & & & \\
Large grain fraction & & & 0.90 \\
Size small & $f_{\text {large }}$ & 0.90 & $0.005-1$ \\
Size large & $\mathrm{a}[\mu \mathrm{m}]$ & $0.005-1$ & \\
Size distribution & $\mathrm{a}[\mu \mathrm{m}]$ & $0.005-1000$ & $0.005-1000$ \\
Composition & & $\mathrm{d} n / \mathrm{d} a \propto a^{-3.5}$ & \\
Total gas-to-dust ratio & $\mathrm{g} / \mathrm{d}$ & $\mathrm{ISM}$ & \\
\hline
\end{tabular}

Notes. Dust composition is taken from Draine \& Lee (1984) and Weingartner \& Draine (2001).

The dust temperature and the radiation field are calculated using the continuum ray-tracing module of DALI. Figure A.1 shows the density, dust temperature, radiation field and fractional abundance of CO for the DALI models. The CO abundance map does not include the effects of the grain surface chemistry discussed in this paper.

\section{Appendix B: Chemical model}

The abundance evolution is computed by a chemical solver based on its counterpart in the DALI code (Bruderer et al. 2012; Bruderer 2013). To solve the set of differential equations, CVODE from the SUNDAILS suite is used (Hindmarsh et al. 2005). CVODE was chosen over LIMEX, the which normally used in DALI, as CVODE is faster and more stable on the very stiff grain-surface chemistry models as well as being thread safe, enabling the calculation of multiple chemical models in parallel with OpenMP5

\section{B.1. Initial abundances}

Table B.1 shows the input volatile abundances used in our chemical model. These abundances are depleted in Si and $\mathrm{S}$ but not in Fe with respect to solar. Fe will be frozen-out and will not play an active part in the chemistry.

Table B.2 enumerates all the binding energies used in the model. The list is sorted (left to right, top to bottom) according to molecular mass. Binding energies come from the recommended values from Penteado et al. (2017) For $\mathrm{NH}, \mathrm{NH}_{2}, \mathrm{CH}, \mathrm{CH}_{2}$ and $\mathrm{CH}_{3}$, the binding energy was increased compared with the values from Penteado et al. (2017). $\mathrm{NH}$ and $\mathrm{NH}_{2}$ were calculated using the method of Garrod \& Herbst (2006), while for $\mathrm{CH}, \mathrm{CH}_{2}$

\footnotetext{
5 http://wwW.openmp.org/
}

Table B.1. Initial gas phase abundances for the chemical network.

\begin{tabular}{lclc}
\hline \hline Molecule & Abundance & Molecule & Abundance \\
\hline $\mathrm{H}_{2}$ & 0.5 & $\mathrm{He}$ & $9.75(-2)$ \\
$\mathrm{NH}_{3}$ & $1.45(-6)$ & $\mathrm{H}_{2} \mathrm{O}$ & $1.18(-4)$ \\
$\mathrm{CO}$ & $1(-4)$ & $\mathrm{N}_{2}$ & $2(-5)$ \\
$\mathrm{Si}$ & $4(-10)$ & $\mathrm{CH}_{3} \mathrm{OH}$ & $1(-6)$ \\
$\mathrm{H}_{2} \mathrm{~S}$ & $1.91(-8)$ & $\mathrm{CO}_{2}$ & $1(-5)$ \\
$\mathrm{Fe}$ & $4.27(-7)$ & Grains & $2.2(-12)$ \\
\hline
\end{tabular}

and $\mathrm{CH}_{3}$ the binding energy are calculated by linearly scaling between the values for $\mathrm{C}$ and $\mathrm{CH}_{4}$ to the number of hydrogen atoms.

\section{B.2. $\mathrm{H}_{2}$ formation rate}

The formation of $\mathrm{H}_{2}$ is implemented following Cazaux \& Tielens (2004), who give the $\mathrm{H}_{2}$ formation rate as (in $\mathrm{H}_{2}$ molecules per unit volume per unit time):

$R_{\mathrm{H}_{2}}=\frac{1}{2} n_{\mathrm{H}} v_{\mathrm{H}} N_{\text {grain }} \pi a_{\text {grain }}^{2} \epsilon_{\mathrm{H}_{2}} S_{\mathrm{H}}(T)$,

where $n_{\mathrm{H}}$ is the number density of gaseous $\mathrm{H}, v_{\mathrm{H}}$ is the thermal velocity of atomic hydrogen, $N_{\text {grain }}$ is the absolute number density of grains, $a_{\text {grain }}$ is the grain radius. $S_{\mathrm{H}}(T)$ is the sticking efficiency (Cuppen et al. 2010), given by

$S_{\mathrm{H}}(T)=\frac{1}{1.0+0.04 \sqrt{T_{\text {gas }}+T_{\text {dust }}}+2 \times 10^{-3} T_{\text {gas }}+8 \times 10^{-6} T_{\text {gas }}^{2}}$. 
A. D. Bosman et al.: CO destruction in protoplanetary disk midplanes

Table B.2. Binding energies for all the species in the chemical network.

\begin{tabular}{|c|c|c|c|c|c|c|c|}
\hline Molec. & $E_{\text {bind }}(\mathrm{K})$ & Molec. & $E_{\text {bind }}(\mathrm{K})$ & Molec. & $E_{\text {bind }}(\mathrm{K})$ & Molec. & $E_{\text {bind }}(\mathrm{K})$ \\
\hline $\mathrm{H}$ & 600 & $\mathrm{H}_{2}$ & 430 & $\mathrm{He}$ & 100 & $\mathrm{C}$ & 800 \\
\hline $\mathrm{CH}$ & 873 & $\mathrm{CH}_{2}$ & 945 & $\mathrm{~N}$ & 800 & $\mathrm{CH}_{3}$ & 1018 \\
\hline $\mathrm{NH}$ & 1577 & $\mathrm{CH}_{4}$ & 1090 & $\mathrm{NH}_{2}$ & 2354 & $\mathrm{O}$ & 800 \\
\hline $\mathrm{NH}_{3}$ & 3130 & $\mathrm{OH}$ & 2850 & $\mathrm{H}_{2} \mathrm{O}$ & 5770 & $\mathrm{C}_{2}$ & 1600 \\
\hline $\mathrm{C}_{2} \mathrm{H}$ & 2137 & $\mathrm{C}_{2} \mathrm{H}_{2}$ & 2587 & $\mathrm{CN}$ & 1600 & $\mathrm{C}_{2} \mathrm{H}_{3}$ & 3037 \\
\hline $\mathrm{HCN}$ & 3610 & HNC & 2050 & $\mathrm{C}_{2} \mathrm{H}_{4}$ & 3487 & $\mathrm{CO}$ & 855 \\
\hline $\mathrm{H}_{2} \mathrm{CN}$ & 2400 & $\mathrm{~N}_{2}$ & 790 & $\mathrm{Si}$ & 2700 & $\mathrm{C}_{2} \mathrm{H}_{5}$ & 3937 \\
\hline $\mathrm{CH}_{2} \mathrm{NH}$ & 3428 & $\mathrm{HCO}$ & 1600 & $\mathrm{SiH}$ & 3150 & $\mathrm{C}_{2} \mathrm{H}_{6}$ & 2300 \\
\hline $\mathrm{H}_{2} \mathrm{CO}$ & 2050 & NO & 1600 & $\mathrm{SiH}_{2}$ & 3600 & $\mathrm{CH}_{2} \mathrm{OH}$ & 4330 \\
\hline $\mathrm{CH}_{3} \mathrm{O}$ & 2655 & HNO & 2050 & $\mathrm{SiH}_{3}$ & 4050 & $\mathrm{CH}_{3} \mathrm{OH}$ & 4930 \\
\hline $\mathrm{O}_{2}$ & 1000 & $\mathrm{~S}$ & 1100 & $\mathrm{SiH}_{4}$ & 4500 & HS & 1500 \\
\hline $\mathrm{O}_{2} \mathrm{H}$ & 3650 & $\mathrm{H}_{2} \mathrm{O}_{2}$ & 5700 & $\mathrm{H}_{2} \mathrm{~S}$ & 2743 & $\mathrm{C}_{3}$ & 2400 \\
\hline $\mathrm{C}_{3} \mathrm{H}$ & 2937 & $\mathrm{C}_{2} \mathrm{~N}$ & 2400 & $\mathrm{C}_{3} \mathrm{H}_{2}$ & 3387 & $\mathrm{H}_{2} \mathrm{CCC}$ & 2110 \\
\hline $\mathrm{CH}_{2} \mathrm{CCH}$ & 3837 & $\mathrm{HCCN}$ & 3780 & $\mathrm{C}_{2} \mathrm{O}$ & 1950 & $\mathrm{CH}_{2} \mathrm{CCH}_{2}$ & 4287 \\
\hline $\mathrm{CH}_{2} \mathrm{CN}$ & 4230 & $\mathrm{CH}_{3} \mathrm{CCH}$ & 4287 & $\mathrm{SiC}$ & 3500 & $\mathrm{CH}_{2} \mathrm{CN}$ & 4680 \\
\hline $\mathrm{HC}_{2} \mathrm{O}$ & 2400 & $\mathrm{HCSi}$ & 1050 & $\mathrm{CH}_{2} \mathrm{CO}$ & 2200 & $\mathrm{CH}_{3} \mathrm{CHCH}_{2}$ & 5187 \\
\hline $\mathrm{CNO}$ & 2400 & $\mathrm{NH}_{2} \mathrm{CN}$ & 1200 & $\mathrm{OCN}$ & 2400 & $\mathrm{SiCH}_{2}$ & 1100 \\
\hline $\mathrm{SiN}$ & 3500 & $\mathrm{CH}_{3} \mathrm{CO}$ & 2320 & HCNO & 2850 & $\mathrm{HNCO}$ & 2850 \\
\hline HNSi & 1100 & $\mathrm{HOCN}$ & 2850 & HONC & 2850 & $\mathrm{SiCH}_{3}$ & 1150 \\
\hline $\mathrm{CH}_{3} \mathrm{CHO}$ & 3800 & $\mathrm{CO}_{2}$ & 2990 & $\mathrm{CS}$ & 1900 & $\mathrm{~N}_{2} \mathrm{O}$ & 2400 \\
\hline $\mathrm{SiO}$ & 3500 & $\mathrm{COOH}$ & 5120 & $\mathrm{HCS}$ & 2350 & $\mathrm{C}_{2} \mathrm{H}_{5} \mathrm{OH}$ & 5200 \\
\hline $\mathrm{CH}_{3} \mathrm{OCH}_{3}$ & 3300 & $\mathrm{H}_{2} \mathrm{CS}$ & 2700 & $\mathrm{H}_{2} \mathrm{SiO}$ & 1200 & $\mathrm{HCOOH}$ & 5000 \\
\hline $\mathrm{NO}_{2}$ & 2400 & NS & 1900 & $\mathrm{C}_{4}$ & 3200 & SO & 2600 \\
\hline $\mathrm{C}_{4} \mathrm{H}$ & 3737 & $\mathrm{C}_{3} \mathrm{~N}$ & 3200 & $\mathrm{C}_{4} \mathrm{H}_{2}$ & 4187 & $\mathrm{C}_{4} \mathrm{H}_{3}$ & 4637 \\
\hline $\mathrm{HC}_{3} \mathrm{~N}$ & 4580 & $\mathrm{HNC}_{3}$ & 4580 & $\mathrm{C}_{3} \mathrm{O}$ & 2750 & $\mathrm{CH}_{2} \mathrm{CHCCH}$ & 5087 \\
\hline NCCN & 1300 & $\mathrm{SiC}_{2}$ & 1300 & $\mathrm{CH}_{2} \mathrm{CHCN}$ & 5480 & $\mathrm{SiC}_{2} \mathrm{H}$ & 1350 \\
\hline $\mathrm{C}_{2} \mathrm{H}_{4} \mathrm{CN}$ & 5930 & $\mathrm{C}_{4} \mathrm{H}_{6}$ & 5987 & $\mathrm{SiC}_{2} \mathrm{H}_{2}$ & 1400 & SiNC & 1350 \\
\hline $\mathrm{C}_{2} \mathrm{H}_{5} \mathrm{CN}$ & 6380 & $\mathrm{C}_{2} \mathrm{~S}$ & 5320 & $\mathrm{Fe}$ & 4200 & $\mathrm{CH}_{3} \mathrm{COCH}_{3}$ & 3300 \\
\hline $\mathrm{CH}_{2} \mathrm{OHCO}$ & 6230 & $\mathrm{COOCH}_{3}$ & 3650 & $\mathrm{C}_{5}$ & 4000 & $\mathrm{CH}_{2} \mathrm{OHCHO}$ & 6680 \\
\hline $\mathrm{CH}_{3} \mathrm{COOH}$ & 6300 & $\mathrm{HCOOCH}_{3}$ & 4000 & OCS & 2888 & $\mathrm{SiO}_{2}$ & 4300 \\
\hline $\mathrm{SiS}$ & 3800 & $\mathrm{C}_{5} \mathrm{H}$ & 4537 & $\mathrm{C}_{4} \mathrm{~N}$ & 4000 & $\mathrm{C}_{5} \mathrm{H}_{2}$ & 4987 \\
\hline $\mathrm{CH}_{3} \mathrm{C}_{4} \mathrm{H}$ & 5887 & $\mathrm{~S}_{2}$ & 2200 & $\mathrm{SO}_{2}$ & 5330 & $\mathrm{SiC}_{3}$ & 1600 \\
\hline $\mathrm{CH}_{3} \mathrm{C}_{3} \mathrm{~N}$ & 6480 & $\mathrm{HS}_{2}$ & 2650 & $\mathrm{SiC}_{3} \mathrm{H}$ & 1650 & $\mathrm{H}_{2} \mathrm{~S}_{2}$ & 3100 \\
\hline $\mathrm{C}_{3} \mathrm{~S}$ & 3500 & $\mathrm{C}_{6}$ & 4800 & $\mathrm{C}_{6} \mathrm{H}$ & 5337 & $\mathrm{C}_{5} \mathrm{~N}$ & 4800 \\
\hline $\mathrm{C}_{6} \mathrm{H}_{2}$ & 5787 & $\mathrm{HC}_{5} \mathrm{~N}$ & 6180 & $\mathrm{SiC}_{4}$ & 1900 & $\mathrm{C}_{6} \mathrm{H}_{6}$ & 7587 \\
\hline $\mathrm{C}_{4} \mathrm{~S}$ & 4300 & $\mathrm{C}_{7}$ & 5600 & $\mathrm{C}_{7} \mathrm{H}$ & 6137 & $\mathrm{C}_{7} \mathrm{H}_{2}$ & 6587 \\
\hline $\mathrm{CH}_{3} \mathrm{C}_{6} \mathrm{H}$ & 7487 & $\mathrm{CH}_{3} \mathrm{C}_{5} \mathrm{~N}$ & 7880 & $\mathrm{C}_{8}$ & 6400 & $\mathrm{C}_{8} \mathrm{H}$ & 6937 \\
\hline $\mathrm{C}_{7} \mathrm{~N}$ & 6400 & $\mathrm{C}_{8} \mathrm{H}_{2}$ & 7387 & $\mathrm{HC}_{7} \mathrm{~N}$ & 7780 & $\mathrm{C}_{9}$ & 7200 \\
\hline $\mathrm{C}_{9} \mathrm{H}$ & 7737 & $\mathrm{C}_{9} \mathrm{H}_{2}$ & 8187 & $\mathrm{CH}_{3} \mathrm{C}_{7} \mathrm{~N}$ & 9480 & $\mathrm{C}_{10}$ & 8000 \\
\hline $\mathrm{C}_{10} \mathrm{H}$ & 8537 & $\mathrm{C}_{10} \mathrm{H}_{2}$ & 8987 & $\mathrm{C}_{9} \mathrm{~N}$ & 8000 & $\mathrm{HC}_{9} \mathrm{~N}$ & 9380 \\
\hline $\mathrm{C}_{1} 1$ & 8800 & & & & & & \\
\hline
\end{tabular}

Furthermore, $\epsilon_{\mathrm{H}_{2}}$ is the $\mathrm{H}_{2}$ recombination efficiency given by

$$
\begin{aligned}
& \epsilon_{\mathrm{H}_{2}}=\left(1+\frac{1}{4}\left(1+\sqrt{\frac{E_{\mathrm{H}_{C}}-E_{S}}{E_{\mathrm{H}_{P}}-E_{S}}}\right)^{2} \exp \left[-\frac{E_{S}}{k T_{\text {dust }}}\right]\right)^{-1} \\
& \times\left[1+\frac{v_{\mathrm{H}_{C}}}{2 F} \exp \left(-\frac{1.5 E_{\mathrm{H}_{C}}}{k T_{\text {dust }}}\right)\left(1+\sqrt{\frac{E_{\mathrm{H}_{C}}-E_{S}}{E_{\mathrm{H}_{P}}-E_{S}}}\right)^{2}\right]^{-1},
\end{aligned}
$$

where $E_{\mathrm{H}_{p}}=600 \mathrm{~K}, E_{\mathrm{H}_{C}}=10000 \mathrm{~K}$ and $E_{S}=200 \mathrm{~K}$ are the energies of a physisorbed $\mathrm{H}\left(\mathrm{H}_{\mathrm{P}}\right)$, chemisorbed $\mathrm{H}\left(\mathrm{H}_{\mathrm{C}}\right)$ and the energy of the saddle point between the previous two, respectively. $F=10^{-10}$ monolayers $\mathrm{s}^{-1}$ is the accretion flux of $\mathrm{H}$ on the ice for the $\mathrm{H}_{2}$ formation.

\section{B.3. Calculation of grain-surface rates}

To calculate the reaction rate coefficients of grain-surface reactions, the mobility of the molecules over the grain-surface needs to be known. This requires knowledge of the vibrational frequency of a molecule in its potential well on the grain-surface (assuming a harmonic oscillator. This is the frequency at which the molecule will attempt displacements:

$v_{\mathrm{X}}=\sqrt{\frac{N_{\text {sites }} E_{\mathrm{X}, \text { bind }}}{2 \pi^{3} a_{\text {grain }}^{2} m_{X}}}$,

where $N_{\text {sites }}$ is the number of adsorption sites per grain, $E_{\mathrm{X}}$, bind the binding energy of the molecule to the grain, $a_{\text {grain }}=10^{-5} \mathrm{~cm}$ is the grain radius and $m_{X}$ is the mass of the molecule. The hopping rate is the vibrational frequency multiplied by the hopping 
probability, which in the thermal case is:

$R_{\text {hop, X }}=v_{\mathrm{X}} \exp \left(-\frac{f_{\text {diff }} E_{\text {bind, } \mathrm{X}}}{k T}\right)$,

where $k$ is the Boltzmann constant and $T$ is the dust-grain temperature. For atomic and molecular hydrogen, tunnelling is also allowed:

$R_{\text {hop, tunnel, X }}=v_{\mathrm{X}} \exp \left[-\frac{2 a_{\text {tunnel }}}{\hbar} \sqrt{2 \mu f_{\text {diff }} E_{\text {bind, } \mathrm{X}}}\right]$.

For atomic and molecular hydrogen, the fastest of these two rates is used which will almost always be the thermal rate. Only for models with a high $f_{\text {diff }}$, low $a_{\text {tunnel }}$ and low temperature is tunnelling faster than thermal hopping.

The rate coefficient for a grain-surface reaction between species $\mathrm{X}$ and $\mathrm{Y}$ forming product(s) $\mathrm{Z}$ on the grain-surface is given by

$$
\begin{aligned}
k(\mathrm{X}+\mathrm{Y}, \mathrm{Z})= & \min \left[1,\left(\frac{N_{\text {act }}^{2} N_{\text {sites }}^{2} n_{\text {grain }}}{n_{\text {ice }}^{2}}\right)\right] P_{\text {reac }}(\mathrm{X}+\mathrm{Y}, \mathrm{Z}) \\
& \left(\frac{R_{\text {hop, } \mathrm{X}}}{N_{\text {sites }}}+\frac{R_{\text {hop, }, \mathrm{Y}}}{N_{\text {sites }}}\right) \\
= & C_{\text {grain }} P_{\text {reac }}(\mathrm{X}+\mathrm{Y}, \mathrm{Z})\left(R_{\text {hop }, \mathrm{X}}+R_{\text {hop }, \mathrm{Y}}\right)
\end{aligned}
$$

where $N_{\text {act }}=2$ is the number of chemically active layers, $N_{\text {sites }}=$ $10^{6}$ is the number of molecules per ice monolayer, $n_{\text {grain }}=$ $2.2 \times 10^{-12} n_{\text {gas }}$ is the number density of grains with respect to $\mathrm{H}_{2}$ and $n_{\text {ice }}$ is the total number density of species on the grain. $P_{\text {reac }}(\mathrm{X}+\mathrm{Y}, \mathrm{Z})$ is the reaction probability for the reaction. This includes the branching ratios if multiple products are possible as well as a correction for reactions that have a barrier.

The reaction probability is given by

$P_{\text {reac }}(\mathrm{X}+\mathrm{Y}, \mathrm{Z})=b_{r}(\mathrm{X}+\mathrm{Y}, \mathrm{Z}) \exp \left(-\frac{E_{\mathrm{bar}}(\mathrm{X}+\mathrm{Y}, \mathrm{Z})}{k T}\right)$,

where $b_{r}(\mathrm{X}+\mathrm{Y}, \mathrm{Z})$ is the branching ratio, $E_{\mathrm{bar}}(\mathrm{X}+\mathrm{Y}, \mathrm{Z})$ is the energy barrier for the reaction. If either $\mathrm{H}$ or $\mathrm{H}_{2}$ is participating in the reaction, tunnelling is included:

$$
\begin{aligned}
P_{\text {reac, tun }}(\mathrm{X}+\mathrm{Y}, \mathrm{Z}) & =b_{r}(\mathrm{X}+\mathrm{Y}, \mathrm{Z}) \\
& \exp \left(-\frac{2 a_{\text {tunnel }}}{\hbar} \sqrt{2 \mu E_{\mathrm{bar}}(\mathrm{X}+\mathrm{Y}, \mathrm{Z})}\right) .
\end{aligned}
$$

The largest of the tunnelling and thermal crossing probability is taken for the actual rate calculation, for most reactions tunnelling dominates in contrast with hopping, where thermal crossing dominates.

\section{B.4. Implications of modelling assumptions}

\section{B.4.1. Variations in the $\mathrm{H}_{2}$ formation rate}

The formation speed of $\mathrm{H}_{2}$ from atomic hydrogen is very important in our models as, together with the destruction rate of $\mathrm{H}_{2}$, it sets the abundance of atomic hydrogen in the gas. For the formation rate, the prescription of Cazaux \& Tielens (2004) is used. The formalism forces the atomic $\mathrm{H}$ gaseous abundance to $\sim 1 \mathrm{~cm}^{-3}$ for $\zeta_{\mathrm{H}_{2}}=10^{-17} \mathrm{~s}^{-1}$ irrespective of total gas density. A higher or lower atomic hydrogen abundance would strongly affect both the $\mathrm{sCO}+\mathrm{sH}$ route, which would increase in effectiveness with higher atomic hydrogen abundances, and the $\mathrm{sCO}+\mathrm{sOH}$ route, which would decrease in effectiveness with higher atomic hydrogen abundances.

\section{B.4.2. Initial conditions}

The initial conditions for the chemistry are shown in Table. B.1. The focus was on testing the $\mathrm{CO}$ destruction, hence we start with $\mathrm{CO}$ as the major volatile carbon reservoir, with trace amounts of $\mathrm{CO}_{2}(10 \%$ of $\mathrm{CO})$ and $\mathrm{CH}_{3} \mathrm{OH}(1 \%$ of $\mathrm{CO})$. At the densities considered here, there is no strong chemical alteration before freeze-out and desorption are balanced. Furthermore, a small portion of $\mathrm{N}$ is in $\mathrm{NH}_{3}$, with the rest of the nitrogen in $\mathrm{N}_{2}$.

The initial conditions can have a significant impact on the evolution of the $\mathrm{CO}$ abundances. The amount of $\mathrm{H}_{2} \mathrm{O}$ ice on the grains determines the formation rate of $\mathrm{OH}$ on the grain, which in turn impacts the transformation of $\mathrm{CO}$ to $\mathrm{CO}_{2}$. If the chemical evolution is started with a larger portion of carbon in hydrocarbons than assumed in this work, with the excess oxygen put into $\mathrm{H}_{2} \mathrm{O}$, then a shorter $\mathrm{CO}$ destruction timescale will be found in the regions where $\mathrm{CO}_{2}$ formation is effective.

The timescale of the $\mathrm{CO}+\mathrm{He}^{+}$route is especially sensitive to the initial abundances. Starting with a significant fraction of the $\mathrm{CO}$ already incorporated into $\mathrm{CO}_{2}$, such as predicted by Drozdovskaya et al. (2016) and as observed in ices in collapsing envelopes (Pontoppidan et al. 2008), can shorten chemical timescales significantly. Chemical timescales can be further shortened by the removal of $\mathrm{N}_{2}$ from the gas phase, such as by starting the chemical model with $\mathrm{NH}_{3}$ as the dominant nitrogen carrier.

The timescale of the $\mathrm{sCO}+\mathrm{sH}$ route is not very sensitive to the initial abundances, as long as they are molecular. The maximal amount of $\mathrm{CO}$ removal is a function of the amount of $\mathrm{CO}_{2}$ in the ice initially, but as hydrogenation of $\mathrm{CO}$ is at least two orders of magnitude faster than the dissociation of $\mathrm{CO}_{2}$ due to cosmic rays, this means that even when $\mathrm{CO}_{2}$ is the dominant carbon reservoir, the $\mathrm{CO}$ abundance can still be reduced below $10^{-6}$ by $\mathrm{sCO}+\mathrm{sH}$ even with the $\mathrm{CO}$ replenishment.

\section{B.4.3. Chemically active surface}

All our models assume that only the molecules in the top two layers of our ices are participating in chemical reactions. The composition of these layers is assumed to be the same of that of the bulk ice. If the number of layers that can participate in chemical reactions is doubled, then the timescales for all grainsurface reactions decrease by a factor of four (see Eq. (B.7)). However, most of our grain-surface reactions are not limited by the speed of the grain-surface reactions, but by the availability of $\mathrm{H}$-atoms and $\mathrm{OH}$-radicals in the ice. The availability of $\mathrm{H}$-atoms is set by the arrival rate of $\mathrm{H}$-atoms on the grains, and this linearly depends on the total grain-surface but not on the number of chemically active layers. The availability of the $\mathrm{OH}$ radical is primarily set by the destruction rate of $\mathrm{H}_{2} \mathrm{O}$ which is independent of the ice thickness. As such the effect of changing the number of active layers is mostly in setting the exact location of the icelines as a larger number of active layers results in a faster sublimation rate at equal temperatures. Varying the number of active layers between one and eight changes the iceline temperature by less than $1.5 \mathrm{~K}$. This effect is similar to the effect of an one order of magnitude change in the total density.

The total amount of grain-surface, and thus the assummed size of the grains does have some effect on the timescales of $\mathrm{CO}$ destruction. The atomic hydrogen arrival rate on the grain scales with the total available surface, which decreases if the grain size is increased and the gas-to-dust mass ratio is held fixed. However, the $\mathrm{H}_{2}$ formation rate as calculated by Cazaux \& Tielens 


\section{A. D. Bosman et al.: CO destruction in protoplanetary disk midplanes}

(2004) also depends on the total surface area, thus if the total surface area is decreased, that means that the abundance of atomic hydrogen should increase, cancelling the effect of the grain size on the hydrogenation reaction of $\mathrm{CO}$. The production of $\mathrm{CO}_{2}$ actually increases with increasing grain size (and thus decreasing surface area). As the total grain-surface area decreases, a smaller amount of the cosmic-ray-induced UV photons are absorbed by the dust grains, as such the dissociation rate for $\mathrm{H}_{2} \mathrm{O}$ should be slightly higher, creating more $\mathrm{OH}$ radicals and thus increasing the rate of $\mathrm{CO}_{2}$ production.

\section{B.4.4. Mixed ice model}

The code assumes a perfectly mixed ice, that is to say that all species are assumed to be present in the upper chemically active layers at the relative abundances that they are included in the bulk of the ice. Observations of dark clouds indicate that in that stage ice mantles are not perfectly mixed, but have a layered structure (Tielens et al. 1991; Boogert et al. 2015) with a $\mathrm{CO}$-rich layer on top of the $\mathrm{H}_{2} \mathrm{O}$-rich layers. It is expected that ice mantles in protoplanetary disks are also layered. The perfect mixing assumption can have some profound effects on the chemistry of $\mathrm{CO}$. A layered ice below the $\mathrm{CO}$ freeze-out temperature would have a higher fraction of $\mathrm{CO}$ in the surface layers than currently assumed. This would initially speed-up the formation of $\mathrm{CH}_{3} \mathrm{OH}$, however, as the $\mathrm{CO}$ in the top layers is converted into $\mathrm{CH}_{3} \mathrm{OH}$, the rate of $\mathrm{CO}$ destruction would go down. At that point, the $\mathrm{CO}$ destruction rate would be dependent on the $\mathrm{CO}$ exchange rate of the bulk ice and the surface layers which is expected to be slower than the surface diffusion speed. Just above the $\mathrm{CO}$ freeze-out temperature, the top layers of the ice would be made up of mostly $\mathrm{CH}_{3} \mathrm{OH}, \mathrm{CO}_{2}$ and $\mathrm{CH}_{4}$. Since the top layers have very little $\mathrm{H}_{2} \mathrm{O}$, this would lead to very low abundances of $\mathrm{OH}$ radicals in the surface layers and might thus quench the $\mathrm{sCO}+\mathrm{sOH}$ route to form $\mathrm{CO}_{2}$.

Mixing of an ice through bulk diffusion is a slow process. However, in the high density midplanes another process can mix ices: grain-grain collisions. In large parts of the disk, the grain size distribution is in coagulation-fragmentation equilibrium. Especially the destructive collisions leading to cratering and fragmentation should be able to mix up the layered structure.

Multi-phase models that include layering explicitly have been developed (e.g. Taquet et al. 2012; Furuya et al. 2016). Drozdovskaya et al. (2016) compared the results from their twophase (gas and ice) chemical model to the three-phase (gas, surface ice and bulk ice) chemical model from Furuya et al. (2015). They conclude that the two-phase model and the threephase model that includes swapping between bulk and surface ice produces comparable amounts of $\mathrm{CO}_{2}$ in the ice, but that if swapping is excluded, $\mathrm{CO}_{2}$ production is efficiently quenched. More detailed models of disk midplanes that included layering should be done in the future to better quantify the effects of assuming a fully mixed ice on the results presented here.

\section{B.4.5. CO binding energy}

The binding energy of CO depends strongly on the surface on which it is frozen (Collings et al. 2003; Acharyya et al. 2007). Here the binding energy of $\mathrm{CO}$ on a pure $\mathrm{CO}$ ice surface is used, which is applicable to the cold $(<20 \mathrm{~K})$ regions, where $\mathrm{CO}$ is primarily in the ice. In warmer regions however, $\mathrm{CO}$ will mostly be bound to $\mathrm{CO}_{2}, \mathrm{CH}_{3} \mathrm{OH}$ and $\mathrm{H}_{2} \mathrm{O}$, slightly increasing the binding energy of CO (Collings et al. 2003; Acharyya et al. 2007; Noble et al. 2012). The higher binding energy, and thus slower CO diffusion, moves upwards and broadens the temperature range over which the reaction to form $\mathrm{CO}_{2}$ (Eq. (3)) is efficient. 